\title{
Enfermedad de Dupuytren
}

\section{J. M. Arandes Renú}

Hospital CLINIC. BARCELONA

Correspondencia:

arandes@clinic.ub.es

\section{INTRODUCCIÓN}

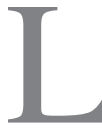

a enfermedad de Dupuytren (ED) es una enfermedad inflamatoria crónica del teno aclaradas, y de transmisión autosómica dominante. Se trata de una fibromatosis aponeurótica superficial, de tipo nodular, caracterizada por una proliferación incontrolada de fibro-miofibroblastos asociada a un aumento de la producción de factores de crecimiento, con abundante síntesis de matriz fibrilar extracelular. La causa que inicia y perpetúa la proliferación tisular es desconocida, pero diversos estudios han encontrado cambios clínicos, bioquímicos, epidemiológicos y morfológicos.

Puede localizarse en diversas partes del organismo, pero la localización más frecuente es en la palma de la mano, en donde afecta a la dermis, a la aponeurosis palmar superficial y a la aponeurosis digital, pudiendo evolucionar hacia un acortamiento longitudinal de sus fibras, lo que ocasiona una flexión irreversible y una impotencia progresiva de los dedos afectos, alterando la función de la mano (Figura 1).

\section{EPIDEMIOLOGÍA}

Existe una significativa variabilidad geográfica, por lo que es difícil poder hacer una simple trasposición de datos de los resultados publicados. La prevalencia varía dependiendo del lugar en el que se tome la muestra de población, y es variable según el sexo y la edad de la población ${ }^{1}$, pues la incidencia es mayor en el hombre y aumenta con la edad ${ }^{2}$.

La ED es una enfermedad de transmisión autosómica dominante con penetración varia$\mathrm{ble}^{3,4}$, con un origen en las razas celtas del norte de Europa. Las migraciones han diseminado la ED por todo el mundo, siendo más frecuente

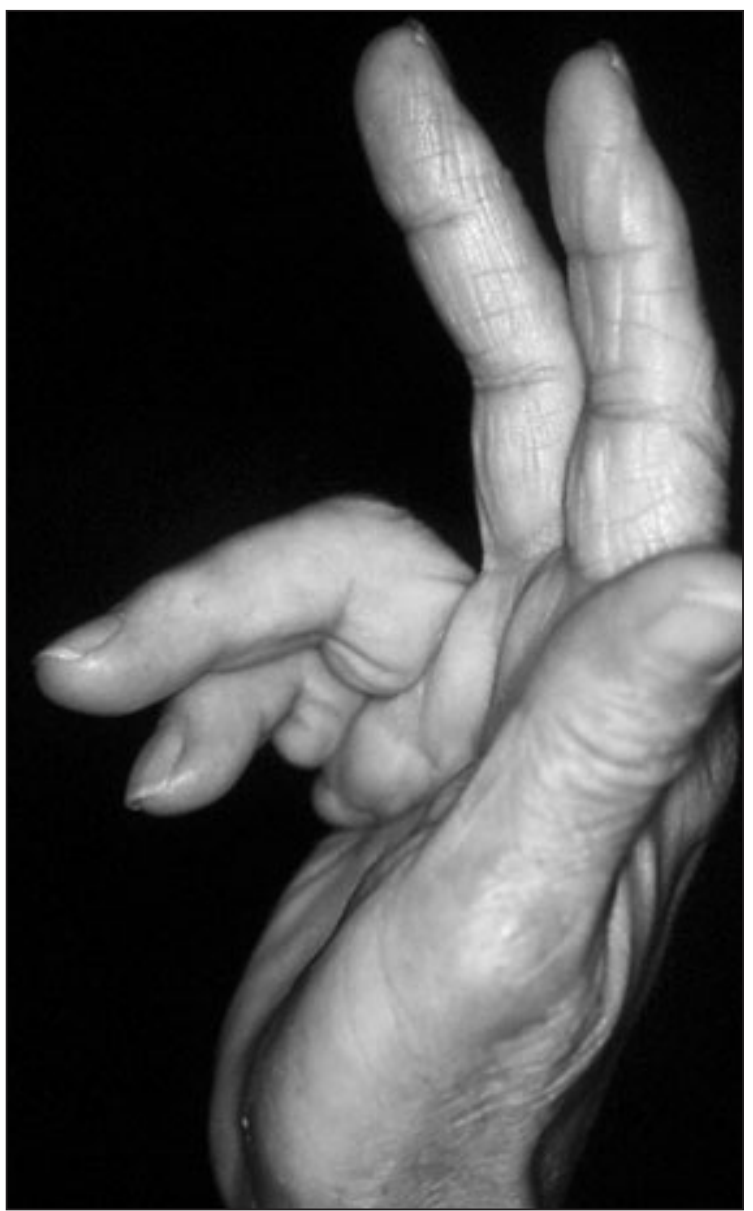

Figura 1: Enfermedad de Dupuytren palmar. 
en Escandinavia, las Islas Británicas, Australia, costa este del norte de América y Japón, la prevalencia decrece en las regiones del Mediterráneo, Sud-América, y se considera rara en África y China 5 .

La prevalencia en la población general es del $3 \%$, y en la población caucasiana entre el 3 y el $6 \% 1,7,8$

En España se han publicado pocos trabajos sobre la prevalencia de la ED. Quintana Guitan ${ }^{9}$ en un trabajo de revisión (casos control), realizado en Zaragoza entre 1964 y 1984 sobre 723 pacientes, estimó una prevalencia del 8,65\%, con una incidencia del $18 \%$ en personas mayores de 75 años.

Rara vez se observa en pacientes menores de 30 años, aunque se han descrito formas el niño, incluso en un niño de 6 meses $^{10}$.

En un estudio de 1.227 pacientes visitados por 108 cirujanos de 12 países realizado por McFarlane ${ }^{11}$ la edad media de aparición es de 49 \pm 12 en el hombre y de $54 \pm 12$ en la mujer.

La proporción hombre/mujer que es de $1 / 8$ alrededor de los cuarenta años, llega al $1 / 2$ en los octogenarios $^{12,13}$, lo que confirma el inicio más tardío de la ED en la mujer ${ }^{1}$.

Entre la mitad y las dos terceras partes de los casos la ED bilateral ${ }^{14,15}$.

\section{FACTORES DE RIESGO ASOCIADOS A LA FRECUENCIA DE APARICIÓN DE LA ED}

\section{Diabetes}

En el estudio de Noble et al. ${ }^{16}$ la incidencia de la ED en diabéticos era del $43 \%$ y del $18 \%$ en el grupo control $(p=0,001)$. La incidencia de la ED en diabéticos varía con la edad y la duración de la diabetes: $30 \%$ en pacientes que padecen diabetes desde hace menos de 5 años y $80 \%$ en los que la han padecido durante más de 20 años ${ }^{17}$.

El aspecto clínico de la ED en los diabéticos es algo diferente, con una mayor afectación del tercer dedo, una evolución más lenta y una localización menos frecuente pero más grave en el $5^{\circ}$ $\operatorname{dedo}^{16,18-20}$.

\section{Epilepsia}

Existen pocas evidencias concluyentes sobre la correlación entre ED y epilepsia. Entre el 2 y el 3\% de los pacientes intervenidos de una ED son epilépticos ${ }^{11,21}$. La frecuencia de ED en pacientes epilépticos adultos varía entre el 10 y el $56 \%$ según las series publicadas, la proporción aumenta con la edad ${ }^{22-26}$.

No se ha demostrado una correlación entre ED y la toma de fenobarbital.

\section{Alcoholismo crónico}

La relación no ha sido establecida, pero diversas publicaciones demuestran un aumento de frecuencia de casos de ED en pacientes alcohólicos ${ }^{27-29}$. Noble et al. ${ }^{30}$ en una revisión bibliográfica con una tabla con la distribución entre la población general, alcohólicos, y pacientes con cirrosis hepática, encontraron que la frecuencia de pacientes alcohólicos y con hepatopatía no alcohólica era más elevada en los pacientes con Dupuytren que en la población general (28 y $22 \%$ respectivamente contra $8 \%$ en el grupo control), pero estas diferencias tenían una significación marginal $(\mathrm{p}<0,05)$; y que la contractura en los pacientes alcohólicos presentaba una mayor gravedad que los pacientes con una hepatopatía no alcohólica. El factor genético era predominante puesto que la cirrosis en pacientes no caucasianos no se acompaña de un incremento de ED. Concluyen que probablemente la incidencia es mayor en los alcohólicos pero que los factores genéticos son los que tienen una mayor importancia etiológica.

\section{Tabaquismo}

An et al. ${ }^{31}$ en un estudio retrospectivo de pacientes fumadores comparados con un grupo control encontraron que el tabaquismo tiene una relación estadísticamente significativa con la $\mathrm{ED}$; que los pacientes intervenidos por una ED son más frecuentemente fumadores $(76,5 \%)$ que los del grupo control $(37,2 \%)$, y que es independiente del sexo. Sugieren que el tabaco ocasiona una oclusión microvascular y una posterior fibrosis y contractura. Seguramente se trata de un factor agravante cuando se asocia a otros factores como el alcohol. 
En el estudio de Reykjavik, publicado por Gudmundsson et al. ${ }^{2}$ con un seguimiento de 1.297 varones durante 15 años, la ED es habitual en los grandes fumadores, de más de 25 cigarrillos/día ( $p=0,018)$. Observaron una mayor mortalidad en pacientes con mayor afectación, estimándose que el $42 \%$ de los fallecimientos eran atribuidos a procesos cancerígenos.

Wilbrand et al. ${ }^{32}$ en un informe sobre la población sueca tras analizar el riesgo de padecer cáncer en 15.212 pacientes intervenidos de ED durante el período 1965-1994 estimaron que el riesgo relativo se incrementaba en un $24 \%$ sobre la población normal, especialmente en los fumadores, con riesgo significativo para neoplasias de boca, esófago, estómago, hígado, páncreas, destacando neoplasias de recto y próstata en varones y de pulmón en mujeres.

\section{Relación con el trabajo y los traumatismos}

Los estudios epidemiológicos permiten deducir tanto que el trabajo manual está asociado con la ED como refutarlo. Pero existe una gran evidencia que demuestra que no hay correlación entre la ocupación y el riesgo de desarrollar una $\mathrm{ED}^{1,7,13,33-36}$

Sólo hay una excepción que es en los trabajadores expuestos a vibraciones en sus manos, pues existe una mayor frecuencia de pacientes con ED que en el grupo control, como se demuestra en el estudio de Cocco et al. ${ }^{37}$ (OR 2,3; IC 95\% 1,5-4,4), y en el de Bovenzi ${ }^{38}$ (OR 2,6; IC $95 \%$ 1,2-5,5). Estos dos estudios evidencian una relación dosis-dependiente aportando un buen soporte a la relación entre la exposición a las vibraciones y la $\mathrm{ED}^{39}$.

La frecuencia de desarrollo o incremento de una ED después de una fractura distal del radio oscila entre el 0 y el $11 \%{ }^{40-46}$, aunque suele afectar a personas mayores y en un grado inicial $(\mathrm{N})$.

Después de una intervención en la mano, lo que representa un traumatismo, en pacientes con nódulos de Dupuytren, se puede observar la progresión de la ED o la aparición en zonas de la mano no afectadas previamente ${ }^{47}$, especialmente en pacientes con acusada diátesis de Dupuytren, lo que es motivo para valorar la indicación quirúrgica en pacientes con poca afectación ${ }^{48}$.

\section{ETIOPATOGENIA}

La etiología y la patogenia de la ED son desconocidas. Seguramente el origen de la ED es multifactorial $^{49}$.

\section{Factor genético}

La ED es una enfermedad hereditaria. Afecta especialmente a personas de descendencia del norte de Europa y, por las migraciones, a sus descendientes. Se han estudiado tres áreas con una incidencia notoriamente elevada como son Edimburgo $^{3}$, Leeds ${ }^{1}$ y Melbourne ${ }^{50}$. Hueston ${ }^{51}$ la denominó enfermedad de los vikingos por coincidir las áreas con mayor prevalencia con sus migraciones.

La incidencia más elevada es en la población de Islandia, en donde la prevalencia de evidencia clínica de la ED es del 19,2\% de los hombres y del $4,4 \%$ de las mujeres ${ }^{2}$.

\section{ANATOMÍA DE LA APONEUROSIS PALMAR}

El conjunto de estructuras aponeuróticas de la cara palmar de la mano y dedos, constituye una unidad, pero para facilitar su descripción se han diferenciado una serie de estructuras o ligamentos, como ya señalaron Legueu y Ju$\operatorname{vara}^{52}$ :

\section{Aponeurosis palmar media}

Tiene dos capas de fibras, las superficiales o aponeurosis palmar superficial, y las profundas o aponeurosis palmar profunda.

\section{A. Aponeurosis palmar media superficial}

Filogenéticamente la aponeurosis palmar es un flexor de la metacarpo-falángica. En el hombre ha perdido su función primitiva para convertirse en un anclaje de la piel palmar, evitando su deslizamiento sobre las estructuras profundas, para ello existen unos tractos conjuntivos verticales de unión entre la cara profunda de la dermis y la cara superficial de la aponeurosis, estos tractos tabican la grasa subdérmica. 
Otras funciones son:

- Protección de los tendones, músculos, vasos y nervios

- Mantener el ahuecamiento de la palma de la mano evitando las fuerzas que tienden a aplanarla, lo que permite utilizar la palma de la mano como un cazo.

- Formación de túneles fibrosos que contribuyen a la orientación y estabilización de los tendones flexores en la línea media del dedo.

Las fibras transversales tienen función de polea tendinosa adicional proximal a la A1. Es una polea funcional. Se ha denominado polea $\mathrm{A} 0$ a la polea formada por las fibras sagitales que continúan al ligamento metacarpo transverso superficial, y que reemplazan parcialmente a la polea A1 cuando ha sido seccionada ${ }^{53}$. La aponeurosis palmar puede ser la localización de un dedo en resorte ${ }^{54}$.

- El ligamento metacarpo transverso superficial o ligamento natatorio, evita la hiperextensión de los dedos.

- Formación de las comisuras interdigitales.

La aponeurosis palmar media superficial se encuentra por debajo de la piel y del tejido celular subcutáneo, está constituida por tejido fibroso; tiene forma triangular, de vértice proximal; ocupa la parte media de la palma, entre las dos eminencias tenar e hipotenar; es la continuación de la aponeurosis antebraquial, o cuando está presente del palmaris longus, que se inserta en este vértice, aunque se trata de estructuras anatómicas diferentes ${ }^{55}$.

Los pacientes con ED tienen con mayor frecuencia palmaris longus con una incidencia estadísticamente significativa ${ }^{56}$. En ausencia de palmaris longus es el flexor carpi ulnaris el que tensa a la aponeurosis palmar.

La aponeurosis palmar media tiene tres tipos de fibras ${ }^{57}$ :

- Longitudinales pretendinosas e intertendinosas

- Verticales o perforantes, que son:

- Superficiales o cutáneas

- Profundas o tabiques paratendinosos

- Transversales:

- Lig. palmar transverso
- Lig. metacarpo transverso superficial o lig. natatorio

- Lig. metacarpo transverso profundo o lig. intermetacarpiano

\section{a. Fibras longitudinales pretendinosas $e$ intertendinosas (Figura 2A)}

Las fibras longitudinales son bandas pretendinosas divergentes en sentido distal que discurren por encima de los tendones flexores de los dedos, adoptando una forma triangular, son superficiales con respecto a las fibras transversales. Legueu y Juvara ${ }^{52}$ las dividen en dos grupos: las bandas paratendinosas y las intertendinosas. Las bandas paratendinosas son cinco, cuatro para los dedos trifalángicos y una quinta para el pulgar situada ligeramente radial con respecto al primer radio; su cara superficial o ventral está firmemente adherida a la cara profunda de la dermis por tractos fibrosos o fibras verticales. Las fibras intertendinosas también son fibras longitudinales.

Distalmente las fibras longitudinales terminan en la cara profunda de la dermis entre el pliegue palmar distal y los pliegues de flexión digitales proximales; un número menor de fibras termina a ambos lados de las articulaciones metacarpo-falángicas (MCF), unas en la parte profunda de la cresta comisural y otras en la aponeurosis palmar profunda; otras fibras se continúan por los dedos contribuyendo a la formación de la banda retrovascular de la aponeurosis digital ${ }^{58}$. Algunas fibras pretendinosas terminan en la piel

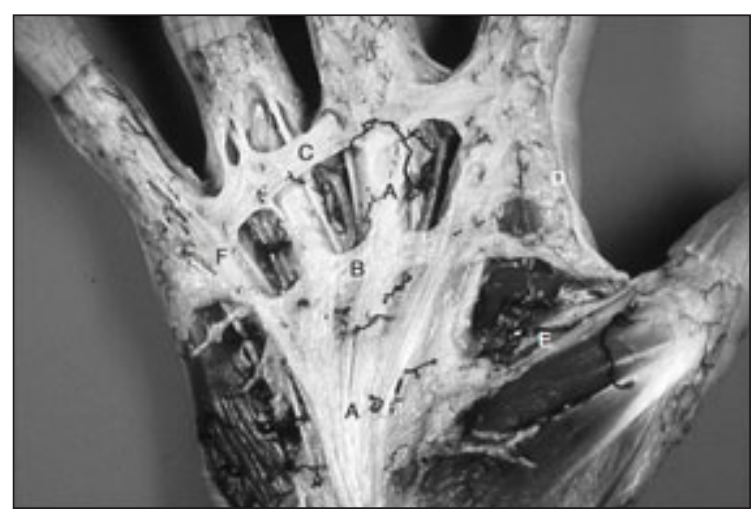

Figura 2: Fibras longitudinales pretendinosas y fibras transversales. A: Fibras pretendinosas. B: Lig. palmar transverso. C: Lig. metacarpo transverso superficial o lig. natatorio. D: Lig. comisural. E: Fibras pretendinosas del pulgar. F: Cuerda pretendinosa del $5^{\circ}$ dedo. 
ventral de la primera falange y llegan hasta el pliegue cutáneo de la articulación interfalángica proximal (IFP) $)^{52,59,60}$.

Las bandas pretendinosas no se continúan con la aponeurosis digital como ha demostrado Gosset $^{61}$. Aunque McGrouther ${ }^{62}$ encuentra que si bien algunas fibras longitudinales no pasan del pliegue de flexión de la MCF y se insertan en el vaina tendinosa o en la piel otras llegan hasta la primera falange. Según Tonkin et al. ${ }^{63}$ se insertan en la polea tendinosa A2.

Las bandas pretendinosas a nivel del cuello de los metacarpianos se dividen en dos bandas en $U$, que se hacen sagitales para alcanzar las caras laterales de la cápsula de las articulaciones IFP, pasando por debajo del ligamento metacarpo transverso superficial o natatorio, y cuando está afectadas por la ED toman la forma de V, y la retracción dificulta la abducción de los dedos vecinos por la doble terminación de la aponeurosis palmar ${ }^{64}$ (Figura 3).

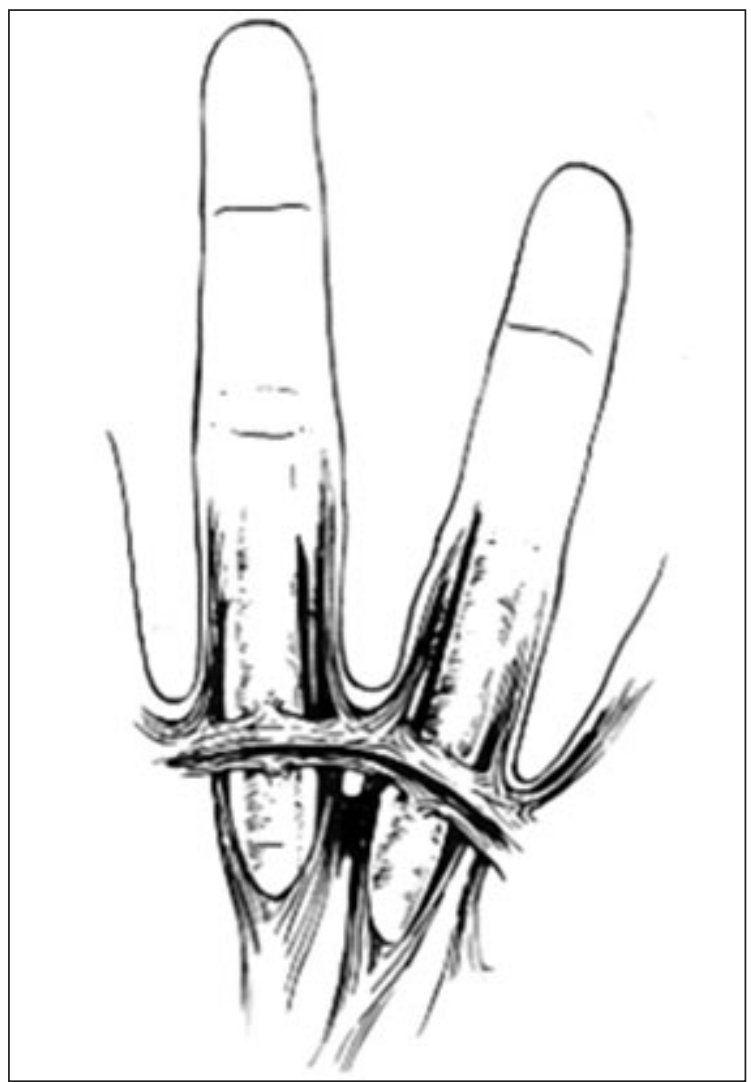

Figura 3: Terminación en U de las fibras pretendinosas. Tomado de McFarlane64.

\section{b. Fibras verticales}

Las fibras verticales, que son más densas a nivel de los pliegues palmares, se extienden desde la dermis hacia la profundidad, atraviesan las fibras longitudinales y transversales y llegan a las vainas de los flexores y a los metacarpianos ${ }^{62}$. Se pueden dividir en superficiales, que fijan la piel, y profundas que fijan a la aponeurosis superficial.

\section{Fibras verticales superficiales}

Las fibras verticales de las aponeurosis se extienden desde la capa superficial de las fibras longitudinales hasta las capas profundas de la piel mezclándose con el tejido fibroso subcutáneo (Figura 4). Esta disposición vertical se observa especialmente en la aponeurosis palmar media, a nivel de los pliegues de flexión y a nivel de los dedos con los ligamentos digitales de Cleland y Grayson. Al fijar la piel la estabilizan evitando que se deslice sobre el plano óseo. En los puntos en que se fija a la piel es donde se forman los nódulos ${ }^{62}$.

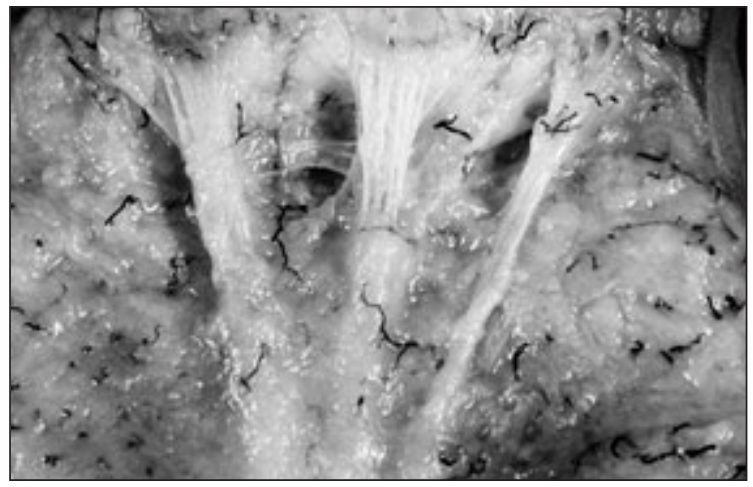

Figura 4: Fibras verticales superficiales.

\section{Fibras verticales profundas}

La cara profunda de las fibras longitudinales está unida a la cara superficial de la aponeurosis profunda, a nivel del pliegue palmar distal, por medio de los tabiques paratendinosos de Legueu y Juvara ${ }^{52}$. Son ocho tabiques colocados sagitalmente, seis intermediarios localizados a los lados de los tendones, y dos marginales, uno radial al flexor del segundo dedo y otro cubital al flexor del quinto dedo. Delimitan siete com- 
partimentos separados, cuatro contienen a los tendones flexores (denominados canales palmares tendinosos) y tres a los mm. lumbricalis (canales lumbricales) acompañados de los vasos y nervios interdigitales (Figura 5). Los elementos vásculo-nerviosos radiales del índice pasan por la región tenar, y los cubitales del meñique por la región hipotenar (el nervio y la arteria digitales cubitales del quinto dedo no están recubiertos por la aponeurosis palmar media); el septo marginal radial envuelve al $\mathrm{m}$. adductor pollicis y al primer $\mathrm{m}$. interossei dorsal, formando el límite volar y radial del canal lumbrical del dedo índice; el septo marginal cubital está fijado a la diáfisis del quinto metacarpiano. Distalmente al canal carpiano esta perforado por el ramo digital del nervio cubital y de la arteria cubital. El límite proximal de los tabiques de Legueu y Juvara no pasa de la arcada palmar y del origen de los $\mathrm{mm}$. lumbricalis.

Si bien se considera que los tabiques paratendinosos no se afectan en la ED, se han descrito algunos casos en los que sí estaban afec$\operatorname{tados}^{65}$.

El extremo distal de los canales tendinosos forma arcadas. Unas mantienen a los tendones flexores en la línea media de los dedos, y junto con la polea A1 impiden que el tendón se separe del plano óseo y evitan el desplazamiento lateral; por otras pasan los elementos vásculo-nerviosos (Figura 6). A nivel de estas arcadas existe una imbricación compleja entre los elementos longitudinales de la aponeurosis palmar, del ligamento metacarpo transverso superficial y de las bandas digitales laterales. A esta arcada Gosset ${ }^{61}$ la denominó quiasma fibroso comisural, y es el punto en el que se unen el septo de Legueu y Juvara, las

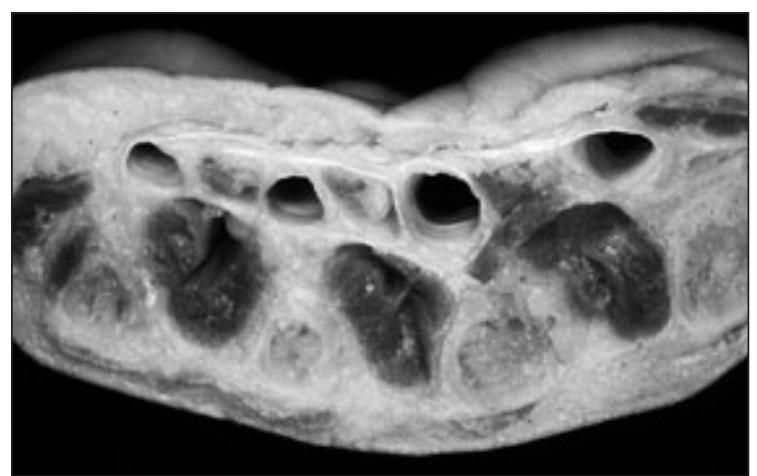

Figura 5: Fibras verticales profundas. Tabiques de Legueu y Juvara ${ }^{52}$.

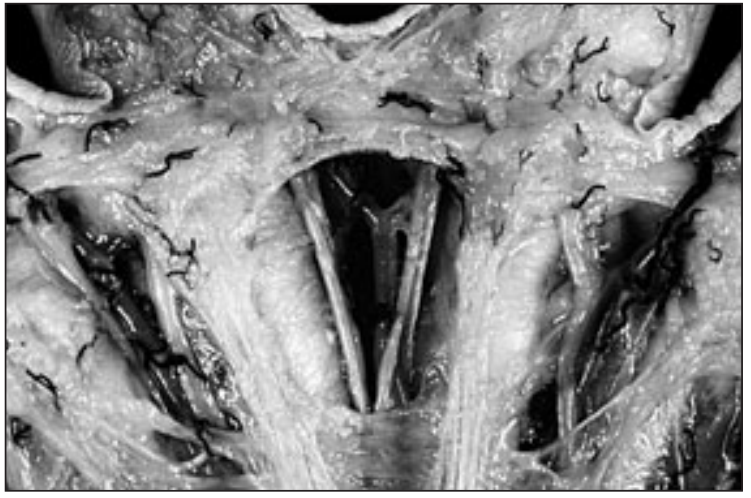

Figura 6: Canales tendinosos y canales lumbricales.

fibras pretendinosas longitudinales en el punto de su bifurcación, la inserción proximal de la banda digital lateral (las bandas digitales laterales nacen de este punto y de la comisura) ${ }^{66}$, las fibras superficiales que se adhieren a la piel y las fibras profundas que se fijan a la vaina del flexor. Se trata de una estructura tridimensional (Figura 7). Esta zona tiene que ser disecada para poder conseguir la extensión del dedo.

A nivel del índice las fibras longitudinales se insertan en la palma proximalmente a la $\mathrm{MCF}^{62}$, y frecuentemente se insertan en la piel del borde radial de la mano, lo que explicaría que la retracción del índice y del pulgar son menos frecuentes y moderadas ${ }^{64}$.

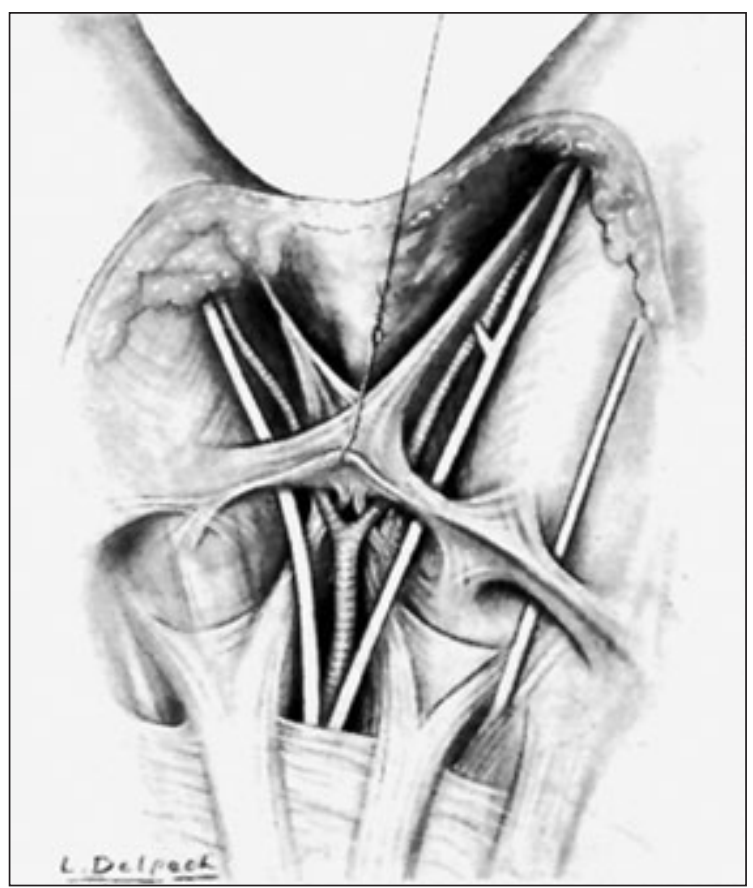

Figura 7: Quiasma fibroso comisural de Gosset ${ }^{91}$.

Revista Iberoamericana de Cirugía de la Mano 


\section{c. Fibras transversales}

Las fibras transversales superficiales son de dos tipos: unas proximales que forman el ligamento palmar transverso y otras fibras distales situadas a nivel de las bases de las falanges que forman el ligamento metacarpo transverso superficial o ligamento natatorio. La formación transversa distal profunda es el ligamento metacarpo transverso profundo o intermetacarpiano, que en realidad es un ligamento interglenoideo.

\section{Ligamento palmar transverso}

Identificado por Albinus ${ }^{67}$, aunque se conoce como ligamento de Skoog ${ }^{68-69}$. Es una banda fibrosa de aproximadamente $1 \mathrm{~cm}$ de ancho, situada a nivel del pliegue cutáneo palmar distal; es más profundo que las fibras pretendinosas y no tienen relación entre sí; sólo se fija a la piel por sus extremos (Figura 2B). No suele estar afectado por la ED, pero su prolongación por la primera comisura sí que se afecta en la $\mathrm{ED}^{69}$.

Por su extremo cubital se fija a nivel del meñique a nivel del pliegue cutáneo distal de la palma y por su extremo radial, a nivel del índice, al extremo radial de los pliegues cutáneos palmar proximal y tenar. El extremo radial se extiende hacia la MCF del pulgar reforzando a la aponeurosis tenar.

\section{Ligamento metacarpiano transverso superficial}

Descrito por Albinus ${ }^{67}$. Grapow ${ }^{70}$ lo denominó ligamento natatorio. Se afecta en la ED. Se trata de una estructura compleja que asegura la transición entre la aponeurosis palmar media y las estructuras aponeuróticas distales, pues en él se insertan las fibras longitudinales pretendinosas y paratendinosas y salen las bandeletas digitales hacia los dedos (Figuras 2C y 7).

Se extiende desde el borde radial del índice hasta el borde cubital el $5^{\circ}$ dedo. En la base del $5^{\circ}$ dedo se desdobla para incluir al pedículo vásculo-nervioso del $5^{\circ}$ dedo y al $\mathrm{m}$. abductor digiti minimi al que se fija. En la base del índice se continúa por la primera comisura por el ligamento comisural distal. Su borde distal se continúa con la aponeurosis digital. El ligamen- to metacarpiano transverso superficial no tiene fibras transversales ${ }^{58}$, sus fibras son longitudinales, estudiadas por Gosset ${ }^{71}$ y por Thomine ${ }^{66}$, y que ascienden por las caras laterales de los dedos hasta la F2. Son profundas con respecto al paquete vásculo-nervioso, por ello en la raíz de los dedos el paquete vásculo-nervioso pasa entre el ligamento palmar transverso superficial y las bandeletas retrovasculares ${ }^{66}$. Estas formaciones laterales también reciben fibras profundas desde los septos y desde las bandeletas longitudinales.

\section{Ligamento metacarpiano transverso profundo}

Descrito por Weitbrecht ${ }^{72}$ y estudiado por Zancolli $^{73}$. También se denomina intermetacarpiano, aunque en realidad se trata de un ligamento interglenoideo porque se extiende desde las placas palmares de las articulaciones MCFs de 2 a $5^{\circ}$ dedos. No se afecta en la ED.

\section{B. Aponeurosis palmar tenar}

La aponeurosis palmar tenar es una delgada capa conjuntiva que recubre a la musculatura tenar externa. Ha sido muy bien sistematizada por Tubiana $^{77}$ y por Mackenny ${ }^{74}$. Se pueden diferenciar varios componentes (Figura 8):

- Fibras transversales:

- Lig. comisurales, proximal y distal

- Lig. palmar transverso

- Fibras longitudinales

- Pretendinosas

- Aponeurosis radial lateral

- Fibras verticales

- De fijación de la piel: prevasculares y retrovasculares

Las fibras transversales de la aponeurosis tenar son la continuación del ligamento metacarpiano transverso superficial o natatorio y del lig. palmar transverso. De Frenne ${ }^{75}$ y Tubiana et al. ${ }^{76-}$ ${ }^{77}$ propusieron denominar a la prolongación del lig. metacarpiano transverso superficial o natatorio ligamento comisural, pues es el que forma la primera comisura (Figura 8F). Desde su origen en el ligamento natatorio se divide en dos fascículos, uno distal o ligamento comisural distal, y 


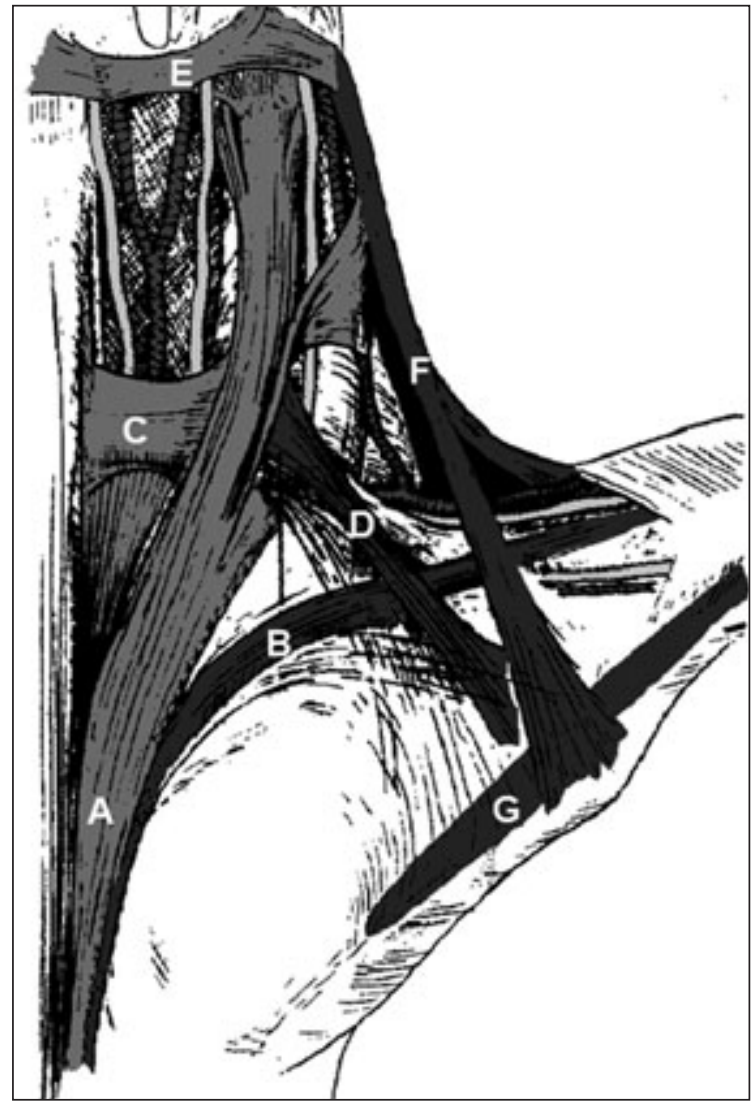

Figura 8: Aponeurosis palmar tenar. Cuerdas radiales. A: Fibras pretendinosas. B: Fibras pretendinosas para el pulgar. C: Lig. palmar transverso. D: Prolongación del lig. palmar transverso hacia el pulgar. E: Lig. metacarpo transverso superficial o lig. natatorio. F: Lig. comisural. G: Aponeurosis radial lateral. Modificado de Tubiana ${ }^{77}$.

otro proximal o ligamento comisural proximal, que se insertan uno a cada lado del tendón del flexor pollicis longus ${ }^{76}$. El lig. palmar transverso llega hasta el pulgar (Figura 8D), y esta prolongación sí que se afecta en la ED.

Las fibras pretendinosas longitudinales, se originan en la aponeurosis palmar media y llegan hasta el sesamoideo cubital del pulgar (Figura 8B).

En la cara lateral del pulgar el esqueleto fibroso forma la aponeurosis radial lateral (Figura 8G), que también se afecta en la ED.

Por el lado cubital la aponeurosis tenar se extiende hacia la profundidad formando el tabique intermuscular externo o tenar, que se une a la aponeurosis del $\mathrm{m}$. adductor pollicis para formar el tabique oblicuo ${ }^{78,79}$ que se fija a la parte proximal de la cresta longitudinal palmar del tercer metacarpiano.

\section{Aponeurosis palmar hipotenar}

Es una delgada capa que recubre a la musculatura hipotenar, está parcialmente cubierta por el m. palmaris brevis.

Reforzada por dos tipos de fibras: longitudinales provenientes de la aponeurosis palmar media, y fibras de fijación del m. palmaris brevis. Tiene escasas fibras verticales de conexión con la piel.

\section{Aponeurosis palmar profunda}

La aponeurosis palmar profunda recubre la cara anterior de la musculatura interósea fijándose a la cara anterior de cada metacarpiano. Su continuidad está interrumpida a nivel del tercer metacarpiano por la inserción del fascículo transverso del $\mathrm{m}$. adductor pollicis ( Figura 9); su cara superficial recibe a los tabiques paratendinosos de la aponeurosis palmar media.

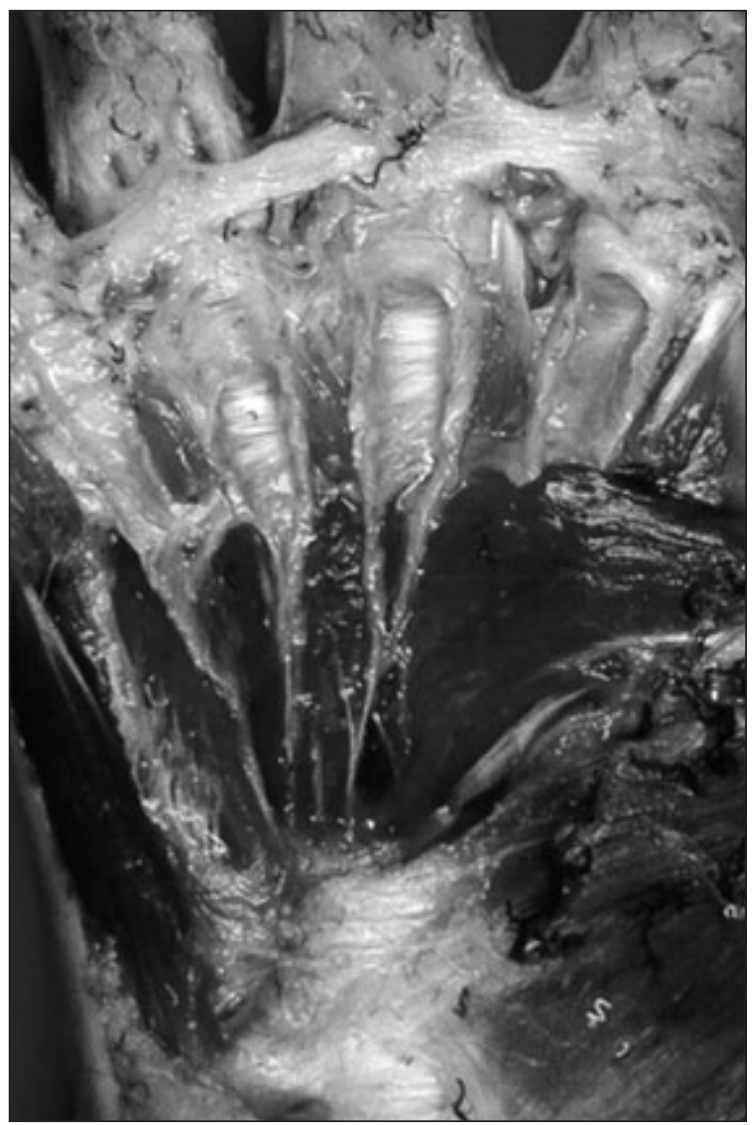

Figura 9: Aponeurosis palmar profunda. Inserción del fascículo transverso del m. adductor pollicis en el III MC 


\section{Aponeurosis digital}

Constituida por:

- Elementos de sostén del aparato extensor, o sistema cutáneo paratendinoso dorsal, o sistema paratendinoso del aparato extensor de Stanisavlejevic y Pool ${ }^{80}$.

- Lig. triangular, banda sagital, lig. retinacular transverso y lig. retinacular oblicuo de Landsmeer

- Ligamentos de sostén cutáneos:

- Lig. de Grayson y de Cleland

- Lig. del nudillo

- Bandas laterales digitales

- Aponeurosis retrovascular de Thomine

Desde la aponeurosis palmar se extiende una banda fibrosa hacia los dedos, formando la aponeurosis digital superficial. Desde los tabiques verticales paratendinosos pasan al interior de los dedos unas prolongaciones que forman la aponeurosis digital profunda ${ }^{81}$.

Las fibras longitudinales que se dirigen a la piel se van reduciendo en cantidad hasta que llegan a la base de los dedos, y unas pocas fibras pueden llegar hasta la piel ventral de F1 y al pliegue de flexión de la IFP ${ }^{52,59}$.

Gosset $^{71}$ basándose en estudios operatorios describe la división de las bandas pretendinosas en dos bandas que llegan a la parte dorsal de la IFP.

Cuando están afectadas por la ED, pueden adoptar dos formas diferentes estudiadas por McFarlane $^{82}$ : las cuerdas laterales y las espiroideas, éstas se colocan alrededor del paquete vascular y del nervio colateral desplazándolos hacia la línea media (Figura 10).

A nivel digital solo se afecta la aponeurosis superficial de los dedos y el ligamento cutáneo de Grayson ${ }^{83}$ mientras que el ligamento cutáneo de Cleland ${ }^{84}$, que es más dorsal no se afecta ${ }^{85}$. Los ligamentos de Grayson y el de Cleland aseguran la estabilidad de la piel de los dedos (Figura 11).

El ligamento retinacular transverso, descrito por Bunnell ${ }^{86}$ como banda transversa de la IFP, es una capa delgada de aponeurosis que se extiende desde la cápsula de la articulación IFP y de la vaina fibrosa de los flexores hasta el borde lateral del aparato extensor, algunas fibras superficiales pasan por encima del tendón para fijarse

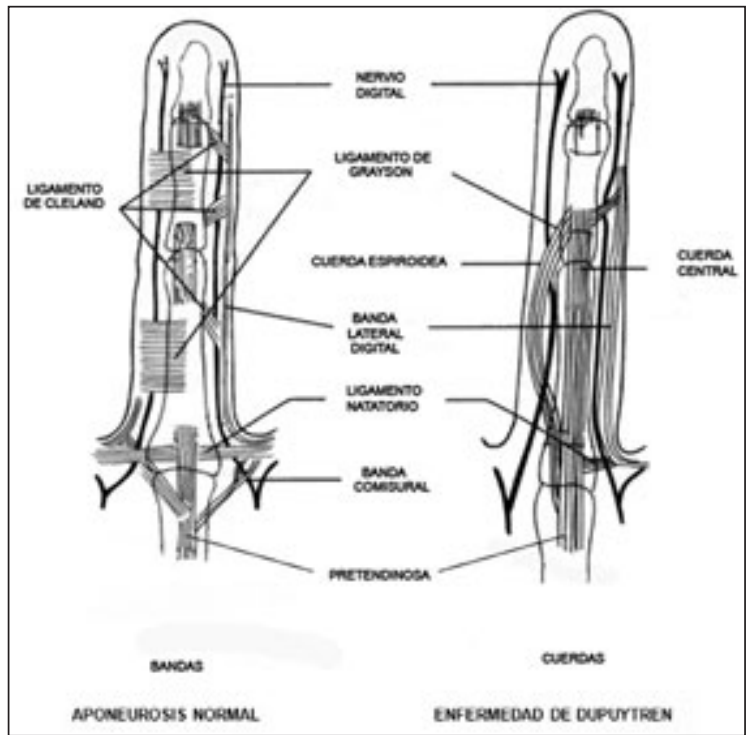

Figura 10: Aponeurosis digital y cuerdas digitopalmares. Modificado de McFarlane ${ }^{64}$.

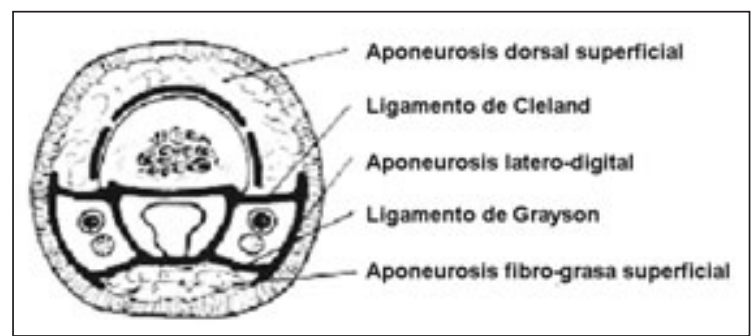

Figura 11: Lig. de Garyson ${ }^{83}$ y de Cleland ${ }^{84}$. Modificado de McFarlane ${ }^{64}$.

con las del otro lado. Al flexionar el dedo, por la salida de los cóndilos, fija al tendón extensor.

El ligamento retinacular oblicuo descrito por Landsmeer ${ }^{87}$ tiene una estructura de aponeurosis, nace de la vaina de los flexores a nivel del cuarto distal de la falange proximal y se dirige distalmente; está recubierto a nivel de la IFD por las fibras del ligamento retinacular transverso al que se une, y finalmente se fija a la cara lateral del tendón extensor, junto al que se inserta en la base de F3. El ligamento retinacular se sitúa palmarmente con respecto al eje de flexión de la IFP $^{88}$. El ligamento contribuye a la extensión de la IFD cuando la IFP está en extensión.

El ligamento retinacular transverso de Bunnell y el ligamento retinacular oblicuo de Landsmeer no se afectan en la ED, pero la retracción de la aponeurosis digital ocasiona una flexión de la IFP que se transmite por medio del ligamento 
retinacular oblicuo a la parte distal del aparato extensor, ocasionando una hiperextensión de la falange distal o deformidad en ojal fija (boutonnière $)^{89}$.

Aponeurosis retrovascular de Thomine ${ }^{89,90}$ : es una banda longitudinal retrovascular, palmar con respecto al ligamento de Cleland, que parte del ligamento natatorio y se fija a la piel, a la falange proximal y a las articulaciones interfalángicas. Gosset $^{71,91}$ lo había denominado lámina digital lateral. Son fibras longitudinales laterales que llegan hasta F3 y que son independientes de la terminación de las bandeletas pretendinosas. Esta estructura es la que forma la aponeurosis retrovascular, que es longitudinal, a diferencia de los ligamentos de Cleland y de Grayson que son transversales. Desde esta aponeurosis es desde donde sale el ligamento retinacular de Landsmeer.

\section{Aponeurosis digital dorsal}

En la parte dorsal de los dedos existe un sistema paratendinoso dorsal. Son estructuras de fijación cutáneas de la piel del nudillo, en donde además de los ligamentos laterales y dorsales de fijación cutánea existen otras fibras de fijación cutánea a las que Milford ${ }^{92}$ denominó fibras cutáneas peritendinosas laterales, que son fibras de unión de la piel dorsal de los dedos con el tendón a nivel de la IFP, y de las bandas laterales digitales o terminaciones de la aponeurosis palmar y que parten del ligamento natatorio.

Para Law y McGrouther ${ }^{93}$ es un sistema de de fibras verticales de fijación de la piel al sistema tendinoso y que a nivel de las articulaciones forman unos tabiques que forman las arrugas de los nudillos, y a los que denominan ligamentos dorsales de la arruga (dorsal wrinkle ligaments). Son los responsables de la forma de los pliegues dorsales del nudillo cuando el dedo está en extensión, y que desaparecen al flexionar el dedo. Tras una artrodesis de la IFP estos pliegues desaparecen.

\section{CUERDAS DIGITALES}

Las cuerdas de los dedos pueden ser dígitopalmares o digitales aisladas. Las cuerdas digitopalmares son de nueva formación, pues anatómicamente no existe una estructura aponeurótica que continúe las bandas pretendinosas hasta el dedo. Las cuerdas dígito-palmares han sido estudiadas por McFarlane ${ }^{17,82}$ dividiéndolas en tres tipos: central, lateral y espiral que terminan en la vaina tendinosa y tienen inserciones distales a la IFP a nivel de la F2. Otra cuerda digito-palmar es la retrovascular. Las cuerdas digitales aisladas no tienen relación con las cuerdas palmares. En el $5^{\circ}$ dedo existe una cuerda específica, la cuerda cubital fijada al tendón del $\mathrm{m}$. abductor digiti minimi, que desplaza siempre al pedículo vásculonervioso a nivel de la base del dedo hacia la línea media; esta cuerda puede ser lateral o espiral, en cuyo caso rodea al pedículo (Figura 12).

En algunos casos se puede observar una cuerda cutánea palmar localizada exclusivamente a nivel de la IFP de los dedos trifalángicos o en la IF del pulgar, que puede ocasionar un déficit de extensión ${ }^{94}$ (Figura 13).

En un mismo paciente pueden encontrarse combinaciones diferentes de las diferentes cuerdas.

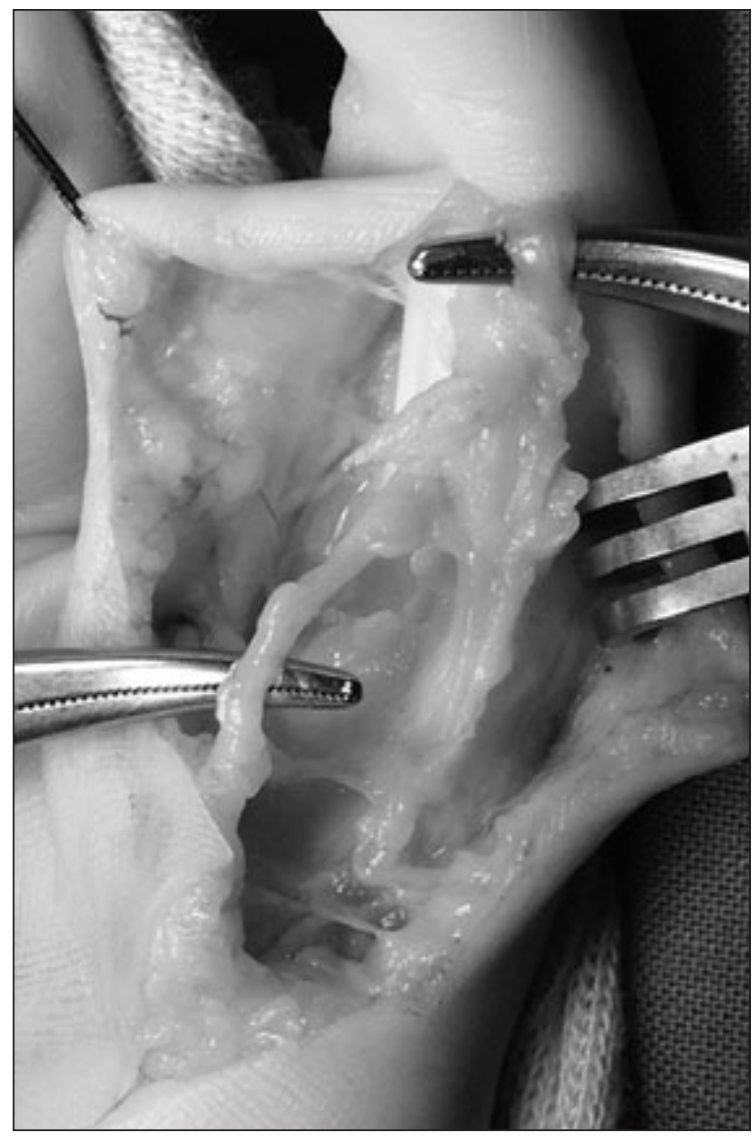

Figura 12: Cuerda espiroidea del abductor digiti minimi. Desplaza al pedículo vásculo-nervioso hacia la línea media. 


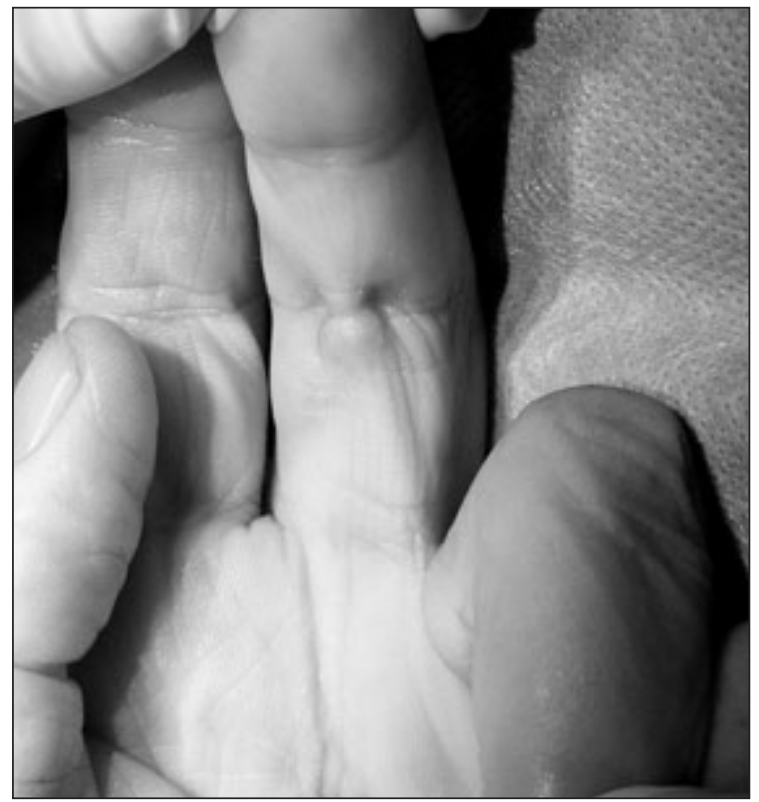

Figura 13: Cuerda palmar aislada de IFP.

\section{ORIGEN DE LA ENFERMEDAD DE DUPUYTREN}

Hay dos teorías sobre el origen de la ED, la intrínseca y la extrínseca:

$1^{a}$ Teoría intrínseca, postulada por Dupuytren, según la cual la causa estaría en la misma aponeurosis palmar, considerando que la enfermedad era una exageración del estado normal de la aponeurosis

$2^{a}$ Teoría extrínseca, desarrollada por Goyrand $^{95} \mathrm{y}$ posteriormente por MacCallum y Hueston ${ }^{94}$ y por Hueston ${ }^{96}$, la lesión primaria (nódulo palmar) asienta en los tejidos superficiales a la aponeurosis, concretamente en la dermis cutánea, desde donde por los septos fibrosos se induce la contractura de la aponeurosis adyacente, y nunca asienta en la cara profunda de la aponeurosis palmar.

Qureshi et al. ${ }^{97}$ examinado histológicamente biopsias de tejidos de la ED y de la piel contigua para evidenciar infiltrados inflamatorios, encuentran, en los nódulos y en las uniones dermoepidérmicas, un incremento del número de células de Langerhans positivas S100 (células epidérmicas de origen dendrítico) y de células CD45-positivas. Proponen que las células de
Langerhans migran desde la epidermis al tejido de Dupuytren, posiblemente como respuesta a cambios locales de los niveles de citoquinas inflamatorias en el tejido. Estos hechos junto a otros trabajos como los de Sugden et al..$^{98}$ sobre el incremento del número de dendrocitos dérmicos (células factor-XIIIa-positivo) y de células inflamatorias en el tejido de la ED sugieren una relación importante entre la piel y la patogénesis de la ED lo que apoya la teoría extrínseca.

Diversos estudios están a favor de la hipoxia localizada a nivel de la aponeurosis como causa de la $\mathrm{ED}^{99}$. La hipoxia ocasiona una necrosis pericitaria seguida de una regeneración. La hipoxia puede favorecer la transformación de pericitos en miofibroblastos ${ }^{100}$. La hipótesis de la hipoxia local puede explicar la elevada frecuencia de ED en los diabéticos, alcohólicos, fumadores y tras un traumatismo ${ }^{101}$.

\section{CLÍNICA}

El diagnóstico de la ED es exclusivamente clínico. Si bien el diagnóstico es fácilmente reproducible en caso de retracción digital, como ha demostrado Lennox et al. ${ }^{102}$, es más difícil en caso de existir únicamente nódulos, incluso para los cirujanos de la mano.

La ED es habitualmente asintomática. De comienzo insidioso, existen varios estadios de la ED. La manifestación inicial consiste en un nódulo, indoloro, que no sigue los movimientos del dedo, localizado a nivel del pliegue palmar distal del dedo anular o del meñique, aunque puede afectar al dedo medio y al pulgar, también se pueden encontrar a nivel de las IFP y de las IFD. El nódulo es la lesión patognomónica de la ED.

En algunos pacientes nunca se producen contracturas, solo nódulos o umbilicaciones, o son muy leves lo que no causa alteraciones funcionales, en otros se forman cuerdas longitudinales que flexionan progresiva, e irreversiblemente, a uno o varios dedos, especialmente el meñique y anular, y menos frecuentemente el índice y el pulgar; la retracción de las fibras longitudinales de la aponeurosis palmar ocasiona una flexión de las MCF y de las IFP, aunque la flexión de la IFP también puede estar ocasionada por cuerdas digitales aisladas sin inserciones en la aponeurosis palmar ${ }^{94}$. 


\section{CLASIFICACIÓN}

Se ha adoptado la clasificación de Tubiana y Michon ${ }^{103}$ que permiten definir con precisión la extensión palmo-digital de la ED. Es un método de cotación numérica que permite a nivel de cada dedo valorar la intensidad de las lesiones.

Se distinguen seis estadios, el 0 es la ausencia de lesión, los otros cinco para los dedos trifalángicos van del grado $\mathrm{N}$ al IV; el $\mathrm{N}$ es la existencia de nódulos palmares o digitales sin flexión, los otros cuatro indican un incremento de $45^{\circ}$ : se calcula sumando el déficit de extensión a nivel de cada una de las tres articulaciones: MCF, IFP, IFD de cada radio ${ }^{104}$. Cuando existe una hiperextensión de la IFD se denomina estadio IV y se indica con una $\mathrm{H}$, en este caso se suman los grados de hiperextensión al déficit de extensión de las otras articulaciones.

Teóricamente para cada dedo trifalángico la deformidad va desde $0^{\circ}$ para la extensión completa, a $200^{\circ}$ (incrustación del dedo en la palma; y de $0^{\circ}$ a $160^{\circ}$ para el pulgar.

La letra $\mathrm{P}$ indica que la lesión es exclusivamente palmar; la $\mathrm{D}$ únicamente digital, la $\mathrm{H}$ indica en las formas avanzadas que la F3 se encuentra en hiperextensión. Cuando la IFP está retraída por encima de $70^{\circ}$ se añade $\mathrm{D}+$.

Pueden diferenciarse seis estadios para los dedos trifalángicos (Figura 14):

0: No existe lesión

$\mathrm{N}$ nódulo, palmar o digital, sin deformidad irreductible

I: la deformación global, suma de las retracciones de las tres articulaciones, está comprendida entre $0^{\circ}$ y $45^{\circ}$

II: $45^{\circ}$ y $90^{\circ}$

III: $90^{\circ}$ y $135^{\circ}$

IV: superior a $135^{\circ}$

A nivel del pulgar Tubiana y Michon ${ }^{103}$ proponen dos tipos de clasificaciones: según la retracción de la primera comisura y según la retracción de la MCF e IF del pulgar (Figuras 15 y 16).

La retracción de la primera comisura la clasifican en cinco estadios correspondientes a pérdidas de $15^{\circ}$ entre el primero y el segundo radios, en comparación con el lado contralateral, normalmente el ángulo es superior a $\operatorname{los} 45^{\circ}$ :
0: ausencia de lesión

$\mathrm{N}$ : nódulos sin retracción

I: apertura de la primera comisura entre $45^{\circ}$ y $30^{\circ}$

II: entre 30 y $15^{\circ}$

III: inferior a $15^{\circ}$

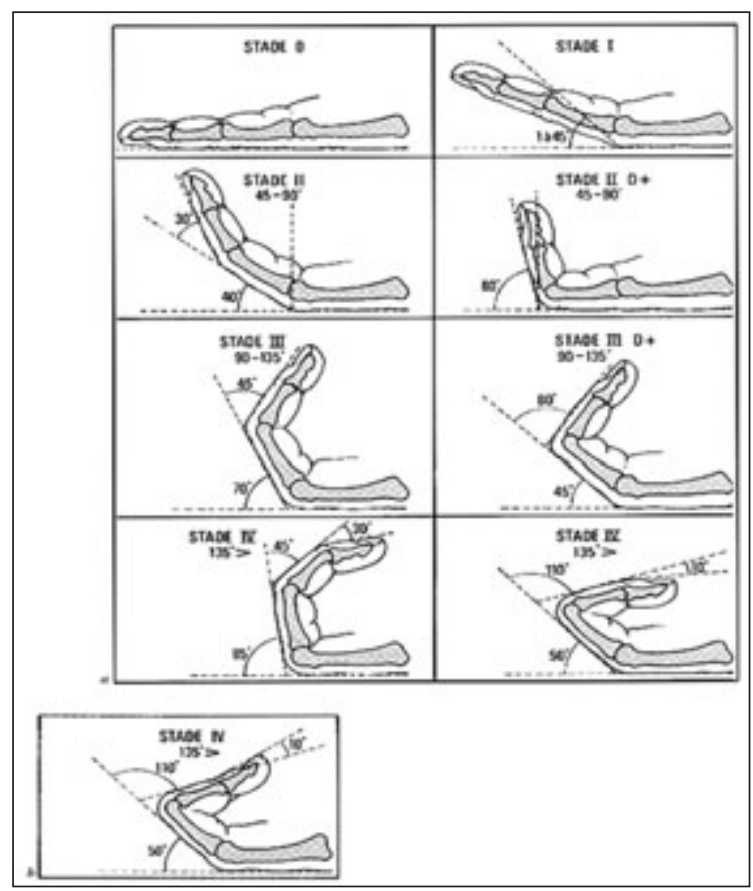

Figura 14: Clasificación de Tubiana y Michon para los dedos trifalángicos ${ }^{103}$.

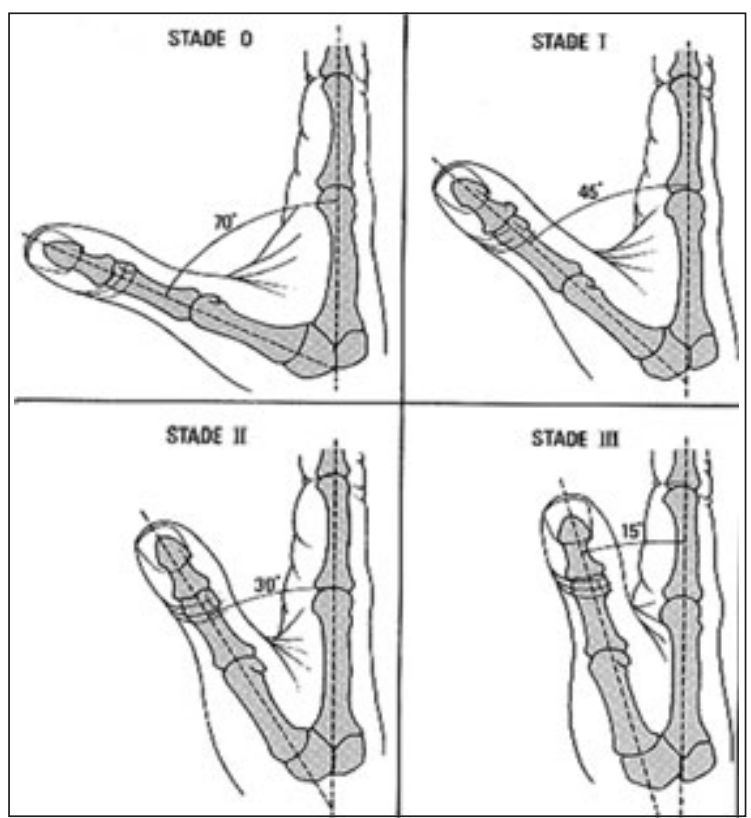

Figura 15: Clasificación de Tubiana y Michon según la retracción de la primera comisura ${ }^{103}$. 


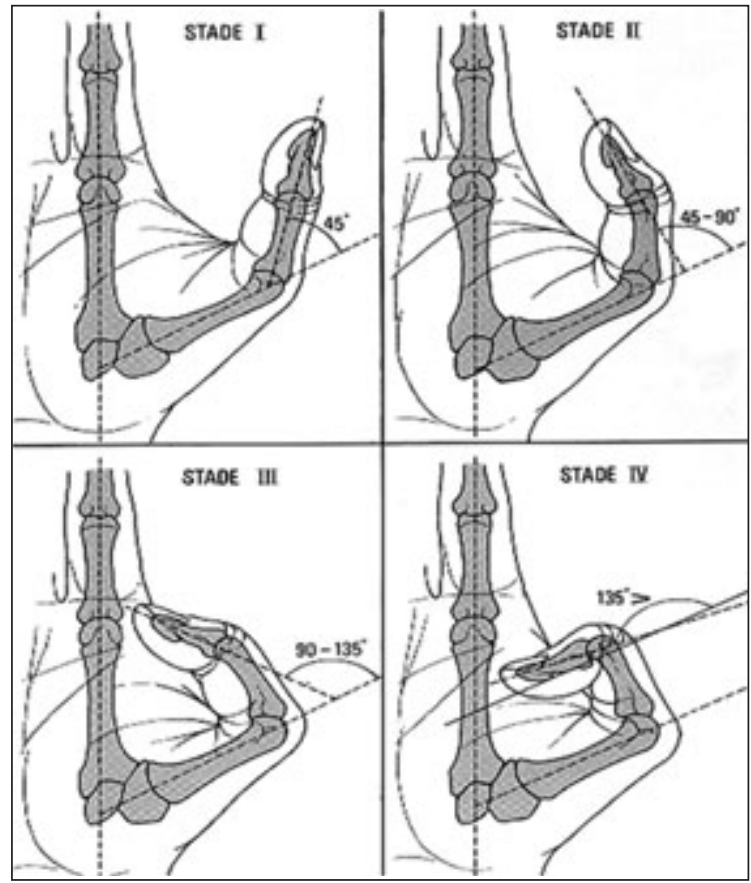

Figura 16: Clasificación de Tubiana y Michon según la retracción de MCF e IF del pulgar ${ }^{103}$.

La clasificación para valorar el déficit de extensión de la MCF e IF del pulgar es igual a la empleada para los dedos trifalángicos, cada estadio corresponde a una progresión de $45^{\circ}$; el resultado estará comprendido entre 0 y $160^{\circ}$.

Esta clasificación permite establecer el estado preoperatorio para poder valorar el resultado postoperatorio y la evolución. Para la valoración de las revisiones de las manos operadas, utiliza otras letras: G para los injertos cutáneos; $\mathrm{R}$ para las recidivas y $\mathrm{E}$ para las extensiones. F señala la rigidez postquirúrgica de los dedos operados, siendo necesario añadir la localización y el grado. A para la amputación y AZ para la artrodesis.

\section{DIAGNÓSTICO DIFERENCIAL}

En la fase de retracción se tiene que hacer el diagnóstico diferencial con otras afecciones:

- la camptodactilia (de kamptós, flexionado, y dáktylos, dedo)

- la garra cubital congénita

- el sarcoma epiteloide de la palma de la mano, tumor maligno raro que afecta a pacientes jóvenes del sexo masculino, lo- calizado en la extremidad distal del miembro superior en forma de nódulos subcutáneos, y que puede localizarse en la palma de la mano ${ }^{105,106}$

\section{DIÁTESIS}

El concepto de diátesis expresa la tendencia o disposición natural a padecer una ED. No es la causa suficiente, pero sí necesaria, para padecer una enfermedad determinada.

La diátesis en la ED es un factor predictivo para la existencia de lesiones más graves, mayor riesgo de recurrencia después del tratamiento quirúrgico, y la existencia de localizaciones extrapalmares.

Los factores clínicos que indican una diátesis en la ED son para Hueston ${ }^{107}$ :

Origen en Europa del Norte (etnia)

Bilateralidad

Antecedentes familiares

Existencia de lesiones ectópicas

Hindocha et al. ${ }^{108}$ añaden otros dos parámetros clínicos: el sexo masculino y comienzo de la ED en una edad joven (antes de los 50 años).

Las formas multifocales de la ED, presentan una fuerte diátesis. En estas formas en un mismo paciente, de forma simultánea o sucesiva, en lugares anatómicos diferentes, se presentan lesiones histológicamente muy similares -proliferación de tejido fibroblástico y producción de colágeno del tipo III- como ocurre con los cojinetes interfalángicos, la induración plástica del pene, y en la afectación de la aponeurosis plantar.

\section{LOCALIZACIONES EXTRAPALMARES DE LA ENFERMEDAD DE DUPUYTREN}

En diversas zonas del organismo se han descrito depósitos de tejido similares a los de la ED palmar. Este tejido es bioquímica e histológicamente igual que el de la enfermedad de localización palmar.

En la revisión de McFarlane ${ }^{17}$ sobre 1.227 pacientes con ED: 
$22 \%$ tenían afectación de los nudillos (cojinetes interfalángicos de Garrod)

$10 \%$ de la aponeurosis plantar (enfermedad de Ledderhose)

$2 \%$ una induración plástica del pene (enfermedad de De La Peyronie)

\section{Localizaciones extrapalmares:}

a) A nivel dorsal de los dedos, lo que se ha denominado ED dorsal:

Los nódulos dorsales de las articulaciones IFP denominados cojinetes interfalángicos o almohadillas de los nudillos, fueron descritos por Sir James Paget en 1875 al describir las bursas anómalas de las IFP ${ }^{109}$, pero fue Archivald E. Garrod quien en 1893 las relacionó con la $\mathrm{ED}^{110}$. Los denominó cojinetes dorsales de los dedos ${ }^{111} \mathrm{y}$ posteriormente Jones ${ }^{112}$ knuckle-pads (Figura 17).

La etiopatogenia es desconocida. Pueden localizarse en uno o varios dedos, son subcutáneos, adheridos a la piel, elásticos, de forma ovoidea, de consistencia dura. Se pueden desplazar por encima del tendón extensor; la piel que las cubre es normal o hiperpigmentada ${ }^{113}$. No limitan la movilidad articular. Rara vez son dolorosos excepto en los periodos de formación o tras traumatismos iterativos. Son más frecuentes en hombres que en mujeres, entre los 40 y 60 años de edad, y no afecta a los dedos de los pies.

Para Caroli et al. ${ }^{113}$ el $81 \%$ de los pacientes con cojinetes interfalángicos tienen una ED bilateral, mientras que la frecuencia de bilateralidad es del $48 \%$ en los que no tienen cojinetes interfalángicos. También encuentran que la presencia de cojinetes está ligada a las formas más

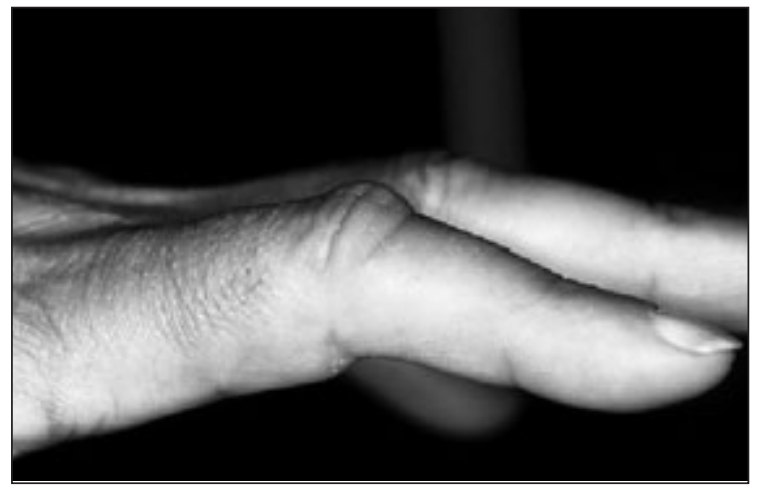

Figura 17: Cojinetes interfalángicos de Garrod $^{110,111}$ o knuckle-pads ${ }^{112}$. evolucionadas de la ED, y frecuentemente están asociados a una enfermedad de Ledderhose o de De La Peyronie.

Mikkelsen ${ }^{114}$ no encontró correlación entre los nódulos y la agresividad clínica de la ED.

El 20\% de los pacientes intervenidos de una ED tienen nódulos interfalángicos, y el $75 \%$ de los pacientes que recidivan tras el tratamiento de una ED tenían cojinetes interfalán$\operatorname{gicos}^{115,116}$.

Nódulos dorsales de la $2^{\mathbf{a}}$ falange. Se sitúan sobre el extensor entre las articulaciones IF según Hueston ${ }^{116}$, y próximos a la IFP para Iselin et al. ${ }^{117}$. Ocasionan un bloqueo en extensión que cede tras la resección de los nódulos.

Deformidad en ojal de F3 (boutonnière), a la que Iselin e Iselin ${ }^{118}$ denominan IV grado de la ED. Es puramente mecánica por la retracción y fibrosis de las bandas laterales y los ligamentos retinaculares. Afecta especialmente del quinto dedo.

\section{b) Otras localizaciones de la ED:}

Los depósitos de fibromatosis en localizaciones diferentes a la mano se conocen como fibromatosis ectópicas. Se han descrito a nivel de la planta del pie, pene, pabellón auricular, lengua, antebrazo, tendón de Aquiles, y en el hueco poplíteo, aunque estas localizaciones son poco frecuentes.

- Fibromatosis de la aponeurosis plantar. Dupuytren en 1831 y 1839 describió por primera vez la enfermedad, su tendencia familiar y la simultaneidad de lesiones a nivel de la palma de la mano y de la planta del pie ${ }^{119,120}$. Se conoce con el epónimo de enfermedad de Ledderhose ${ }^{121}$ por la descripción que hizo el cirujano alemán Georg Ledderhose en 1897.

La lesión es generalmente indolora, y normalmente se presenta en las zonas de la planta que no soportan carga ${ }^{122,123}$, por lo que únicamente precisará tratamiento quirúrgico cuando por su volumen sea incapacitante para la marcha. Aunque se han descrito algunos casos de retracción en flexión del primer dedo ${ }^{124-126}$ no suele ocasionar contracturas de los dedos.

- Lesiones peneanas o enfermedad de De la Peyronie ${ }^{\mathbf{1 2 7}}$. El epónimo se emplea por la descripción de la enfermedad por François Gigot De la Peyronie (1678-1747), en una memoria 
titulada: Mémoires sur quelques obstacles qui s'opposent à l'éjaculation naturelle de la semen$c e$, aportando tres casos de pacientes que presentaban tumores duros en los cuerpos cavernosos, localizados en la cara dorsal del pene, que hacían que el pene se incurvase dorsalmente durante la erección.

- Se ha asociado la ED con la periartritis del hombro (fibromatosis del hombro) ${ }^{22}$. Smith et al. ${ }^{128}$ encuentran 30 casos de ED en 58 pacientes con hombro congelado (52\%), incidencia muy alta si se compara con la de la población general. Comparándola con la incidencia de ED en la población general publicada por Early ${ }^{1}$ tiene una alta significación estadística $(\mathrm{P}<0.001$, test $\left.x^{2}\right)^{128}$.

Se desconoce cuál es la relación entre el hombro congelado y ED, pero las dos entidades tienen algunas similitudes. Histológicamente las dos tienen una disposición del colágeno tipo III en nódulos o cuerdas, con una similar distribución de fibroblastos y miofibroblastos. Las dos presentan una contractura articular y pueden presentarse después de un traumatismo. Existe una alteración metabólica, con un aumento significativo de incidencia de diabetes e hiperlipidemia $^{129}$, y anomalías en las citoquinasas ${ }^{130}$. El hombro congelado presenta alteraciones de la metaloproteinasa matriz y de los inhibidores de la metaloproteinasa matriz ${ }^{128}$.

\section{TRATAMIENTO}

El tratamiento de la ED en su localización palmar tiene tres finalidades, una funcional: corregir las retracciones, y otras profilácticas: evitar las complicaciones y prevenir las recidivas.

No existe un tratamiento curativo, el tratamiento es sintomático y dirigido a corregir las retracciones. Ningún tratamiento consigue evitar el riesgo de recidivas y de extensiones de la ED.

\section{Tratamiento no quirúrgico}

Se han intentado algunos tratamientos por medios físicos: ionización, electrificación, ultrasonidos, terapia con laser, radiaciones, etc., sin éxito.

Naylor et al. ${ }^{131}$ publican una exhaustiva lista de los diversos tratamientos propuestos para la ED:

- Thiosinamina (Rhodallin)

- Thiosinamina-salicilato sódico

- Thiosinamina-yoduro etilico

- Ungüento de Iodina

- Extracto tiroideo

- Solución sulfato de Cooper

- Pepsina

- Extracto paratiroideo

- Ungüento de ácido salicílico al 5\%

- Humanol (grasa humana esterilizada)

- Vitamina E/tocoferoles

- Hidrocortisona

- Tripsina/ $\alpha$ quimotripsina/hialutonidasa

- Paraaminobenzoato de potasio (PABA)

- Dimetilsulfoxido (DMSO)

- Procarbazina (Natulan)

- 6-metil-prednisolona

- Aprotinina (Trasylol)

- $\alpha$-mercaptopropionilglicina (Thiopronin)

- Alopurinol

Todos estos tratamientos no han conseguido prevenir a largo plazo la $\mathrm{ED}^{132}$.

El estudio de la actina del músculo liso del tipo $\alpha$ condujo a Pittet et al. ${ }^{133}$ a realizar un ensayo en fase II con inyecciones intralesionales de $\gamma$-interferon, que es una citoquinasna producida por los linfocitos $\mathrm{T}$ que inhibe a la actina, a los fibroblastos y la producción de colágeno. El estudio no tiene comprobaciones histológicas pero en siete pacientes tratados se obtuvo la disminución del tamaño de los nódulos o desaparecieron en el plazo de tres meses. Recomiendan estudios a largo plazo para validar este tratamiento. Es el tratamiento experimental que parece más prometedor pero el elevado coste del producto hace que su utilización sea limitada ${ }^{134}$.

El tratamiento con colagenasa clostridium histoliticum es el tratamiento actual no quirúrgico que ofrece mejores resultados. Starkweather et al. ${ }^{135}$ son los iniciadores del tratamiento con colagenasa en la ED, al demostrar in vitro que es posible inyectar colagenasa en las cuerdas para fragilizarlas. Conocida la acción de la colagenasa in vitro para valorar su eficacia se realizaron dos estudios denominados CORD (Collagenase Option for the Reduction 
of Dupuytren's). El CORD I estudió la eficacia a los 90 días de la inyección frente a placebo en Estados Unidos ${ }^{136}$ y el CORD II que se realizó en Australia y analiza a 12 meses la eficacia para reducir el grado de contractura y valorar las recurrencias ${ }^{137}$.

Clínicamente se ha determinado que la dosis necesaria de colagenasa para conseguir la ruptura de las cuerdas es de $10.000 \mathrm{U} /$ dosis, equivalente a $0,58 \mathrm{mgr}^{138}$.

Al día siguiente de la inyección de colagenasa en las cuerdas, por maniobras forzadas de extensión, se rompen las cuerdas. A los treinta se consigue una movilidad hasta del $0-5^{\circ}$ de la extensión completa en el $90 \%$ de los pacientes ${ }^{139,140}$. Se pasa de un rango de movimiento de $44^{\circ}$ antes de la inyección a $81^{\circ}$ tras el tratamiento ${ }^{136}$. Los estudios realizados hasta la fecha tienen un corto seguimiento por lo que para confirmar los resultados iniciales son precisos estudios a más largo plazo. La satisfacción de los pacientes es alta del $60 \%$, y el $88 \%$ repetirían el tratamiento para la recurrencia o la progresión de la $\mathrm{ED}^{141}$.

Los reumatólogos franceses iniciaron la técnica de aponeurotomía subcutánea con aguja $^{142-144}$ que consiste en seccionar las cuerdas palmares y/o digitales con la ayuda del bisel de una aguja intradérmica de las empleadas para la anestesia local, inyectándose anestesia local (4cc de xilocaina) junto con $25 \mathrm{mgrs}$ de hidrocortisona (para evitar la reacción inflamatoria). La tasa de recidivas es alta a los cinco años. Esta intervención ha sido preconizada para pacientes de edad avanzada, con una cuerda palmar única y piel no adherida. Tubiana ${ }^{145}$ la propone como primer tiempo en pacientes con graves retracciones de los dedos que ocasionan una maceración de la piel. Está contraindicada a nivel digital por el peligro de lesiones vásculo-nerviosas e incluso tendinosas ${ }^{146}$, y porque generalmente no consigue la extensión de la IFP.

\section{Tratamiento quirúrgico}

La indicación quirúrgica se establecerá cuando estén afectadas las MCF, el test de la mesa (table top test) de Hueston ${ }^{147,148}$ será positivo, o cuando existan grandes nódulos incapacitantes en trabajadores manuales. Smith ${ }^{149}$ estima que la indicación quirúrgica se establace cuando la
MCF esté limitada 30 o más grados, y para Tubiana et al. ${ }^{150}$ y McGrouther ${ }^{151}$ cuando la contractura de las MCF es $\geq 30^{\circ}$, o de $\geq 20^{\circ}$ de las IFP.

La elección de las incisiones dependerá de las necesidades de exposición y del análisis de las opciones para el de cierre cutáneo.

Las técnicas propuestas son:

- Aponeurotomía subcutánea o a cielo abierto

- Aponeurectomía parcial que puede ser selectiva o regional

- Aponeurectomía extensa, total, o radical

- Amputación, de raras indicaciones.

Una vez realizada la aponeurectomía, existen varias opciones en cuanto al tratamiento de la piel:

- Cierre cutáneo mediante sutura, z-plastias, colgajos, o el adelantamiento cutáneo en VY

- Técnica de la palma abierta

- Dermato-aponeurectomía

La dermato-aponeurectomía consiste en resección de la piel y de la aponeurosis afectada seguido de un injerto de piel de grosor total, pues éste inhibe la producción de miofibroblastos. Hueston ${ }^{152}$ presenta un solo caso de recidiva debajo de un injerto de piel total sobre 375 dedos intervenidos y Roy et al. ${ }^{153}$ siete en 100 casos con un seguimiento de 52 meses.

Se ha propuesto la dermato-aponeurectomía para disminuir la tasa de recidivas, especialmente en los casos de tratarse de una forma de ED agresiva $^{154}$, o cuando exista una diátesis especialmente en pacientes jóvenes ${ }^{155}$, o en el tratamiento quirúrgico de las recidivas.

\section{RECIDIVAS-EXTENSIONES}

Recidiva es la reaparición de la ED en las zonas operadas, la extensión sería la aparición de ED en las zonas no operadas, es decir previamente sanas, lo que no se puede prevenir. Ningún tratamiento evita las recidivas.

Las reintervenciones por recidivas son de mayor dificultad y aumentan las complicaciones peroperatorias. 


\section{EVOLUCIÓN}

Es variable, puede evolucionar en meses, o lentamente en años, hay formas de evolución lenta y otras de evolución rápida. En algunos pacientes la lesión avanza progresivamente, en otros existen exacerbaciones y remisiones. La regresión espontánea es rara ${ }^{156}$.
Si bien las recidivas a diez años son frecuentes, y la intervención quirúrgica no puede detener la ED, al menos permite mantener una mano útil, pues los dos tercios de las recidivas no ocasionan alteración funcional, y el tercio restante el estado funcional, con alguna rara excepción, es superior al del preoperatorio ${ }^{157}$.

\section{BIBLIOGRAFÍA}

1. Early PF. Population studies in Dupuytren's contracture. J Bone Joint Surg B, 1962; 44: 602-13.

2. Gudmundsson KG, Arngrímsson R, Sigfússon N, et al. Epidemiology of Dupuytren's disease: clinical, serological, and social assessment. The Reykjavík Study. J Clin Epidemiol, 2000; 53: 291-6.

3. Ling RSM. The genetic factor in Dupuytren's disease. J Bone Joint Surg B, 1963; 45: 709-18.

4. Burge P. Genetics of Dupuytren's disease. Hand Clin, 1999; 15: 63-71.

5. Hindocha S, McGrouther DA, Bayat A. Epidemiological evaluation of Dupuytren's disease. Incidence and prevalence rates in relation to etiology. Hand, online version. htpp://dx.doi. org/10.1007/s11552-0089160-9.

6. Gonzalez SM, Gonzalez RI. Dupuytren's disease. West J Med, 1990; 152: 430-3.

7. Yost J, Winters T, Fett HC Dupuytren's contracture. A statistical study. Amer J Surg, 1955; 90: 568-71.

8. Hueston JT. Dupuytren's contracture. Current Orthop, 1988; 2: 173-178.

9. Quintana Guitián A. Quelques aspects épidémiologiques de la maladie de Dupuytren. Ann Chir Main, 1988; 7: 256-62.

10. Bebbington A, Savage R. Dupuytren's disease in an infant. Case report. J Bone Joint Surg B, 2005; 87: 111-3.

11. McFarlane RM. Epidemiologie de la Maladie de Dupuytren. En: Tubiana R, Hueston JT
(Eds). La Maladie de Dupuytren. Monographie du GEM, Paris: Expansion Scientifique Française, 1986: 106-10.

12. Ross DC. Epidemiology of Dupuytren's disease. Hand Clin, 1999; 15: 53-62.

13. Brenner $P$, Krause-Bergmann A, Van VH. Die Dupuytren-Kontraktur in Norddeutschland. Epidemiologische Erfassungsstudie anhand von 500 Fällen. Unfallchirurg, 2001: 104: 303-11.

14. Bergenudd $H$, Lindgärde $F$, Nilsson BE. Prevalence of Dupuytren's contracture and its correlation with degenerative changes of the hands and feet and with criteria of general health. J Hand Surg B, 1993; 18: 254-7.

15. Brenner $P$, Mailänder $P$, Berger A. Epidemiology of Dupuytren's disease. En: Berger A, Delbrück A, Brenner P, et al. Dupuytren's Disease. Berlin: Springer Verlag, 1994: 244-54.

16. Noble J, Heathcote JG, Cohen $\mathrm{H}$. Diabetes mellitus in the aetiology of Dupuytren's disease. J Bone Joint Surg B, 1984; 66: 322-5.

17. McFarlane RM. Enfermedad de Dupuytren. En: McCarthy JG, May JW, Littler JW (Eds). Cirugía Plástica, vol. 2, La Mano, Buenos Aires: Editorial Médica Panamericana, 1992: 818-51.

18. Hurst LC, Badalamente M. Associated diseases. En: McFarlane RM, McGrouther DA, Flint $\mathrm{MH}$ (Eds). Dupuytren's Disease. Edinburgh: Churchill Livingstone, 1990: 253-60.

19. Renard E, Jacques D, Cham- mas $\mathrm{M}$, et al. Increased prevalence of soft tissue hand lesions in type 1 and type 2 diabetes mellitus: various entities and associated significance. Diabetes Metab, 1994; 20: 513-21.

20. Chammas M, Bousquet $P$, Renard E, et al. Dupuytren's disease, carpal tunnel syndrome, trigger finger, and diabetes mellitus. J Hand Surg A, 1995; 20: 109-14.

21. James JIP. Le caractère génétique de la maladie de Dupuytren et de l'epilepsie idiopathique. En: Tubiana R, Hueston JT (Eds). La Maladie de Dupuytren. Monographie du GEM, Paris: Expansion Scientifique Française, 1986; 84-9.

22. Lund M. Dupuytren's contracture and epilepsy. The clinical connection between Dupuytren's contracture, fibroma plantae, periarthrosis humeri, helodermia, induratio penis plastica and epilepsy, with an attempt at a pathogenic valuation. Acta Psychiatr Neurol Scand, 1941; 16: 465-92.

23. Critchley EMR, Vakil SD, Hayward HW, et al. Dupuytren's disease in epilepsy: result of prolonged administration of anticonvulsivants. J Neurol Neurosurg Psychiatry, 1976; 39: 498-503.

24. Murrell GAC, Hueston JT. Aetiology of Dupuytren's contracture. Aust NZ J Surg, 1990; 60: 247-52.

25. Arafa M, Noble J, Royle SG, et al. Dupuytren's and epilepsy revisited. J Hand Surg B, 1992; 17: 221-4. 
26. McFarlane RM, Ross D. Dupuytren's disease. En: Weinzig J (Ed). Plastic Surgery Secrets. Philadelphia: Hanley and Belfus, 1998: 554-9.

27. Hueston JT. Further studies on the incidence of Dupuytren's contracture. Med J Aust, 1962; 21: 586-8.

28. Su CK, Patek AJ. Dupuytren's contracture: Its association with alcoholism and cirrhosis. Arch Int Med, 1970; 126: 27881.

29. Sabiston DW. Cataracts, Dupuytren's contracture, and alcohol addiction. Am J Ophthalmol, 1973; 76: 1005-7.

30. Noble J, Arafa M, Royle SG, et al. The association between alcohol, hepatic pathology and Dupuytren's disease. J Hand Surg B, 1992; 17: 71-4.

31. An HS, Southworth SR, Jackson WT, et al. Cigarette smoking and Dupuytren's contracture of the hand. J Hand Surg A, 1988; 13: 872-4.

32. Wilbrand S, Ekbom A, Gerdin B. Cancer incidence in patients treated surgically for Dupuytren's contracture. J Hand Surg B, 2000; 25: 283-7.

33. Zachariae L. Dupuytren's contracture. The aetiological role of trauma. Scand J Plast Reconstr Surg, 1971; 5: 116-9.

34. De la Caffinière JY, Wagner R, Etscheid J, et al. Travail manuel et maladie de Dupuytren. Résultats d'une enquête informatisée en milieu sidérurgique. Ann Chir Main, 1983; 2: 66-72.

35. Hueston JT. Editorial: Dupuytren's contracture and occupation. J Hand Surg A, 1987; 12: 657-8.

36. Yi IS, Johnson G, Moneim MS. Etiology of Dupuytren's disease. Hand Clin, 1999; 15: 43-51.

37. Cocco PL, Frau P, Rapallo M, et al. Esposizione professionale a vibrazioni e malattia di Dupuytren: un approccio caso-controllo. Med Lav, 1987; 78: 38692.

38. Bovenzi M, Cerri S, Merseburger A, et al. Hand-arm vibration syndrome and dose-response relation for vibration induced white finger among quarry drillers and stonecarvers. Italian Study Group on Physical Hazards in the Stone Industry. Occup Environ Med, 1994; 51: 603-11.

39. Liss GM, Stock SR. Can Dupuytren's contracture be work-related?: review of the evidence. Am J Ind Med, 1996; 29: 521-32.

40. Bacorn RW, Kurtzke JF. Colles' fracture. A study of two thousand cases from the New York State Workmen's Compensation Board. J Bone Joint Surg A, 1953; 35: 643-58.

41. Frykman G. Fracture of the distal radius including sequelae - Shoulder-hand-finger syndrome, disturbance in the distal radio-ulnar joint and impairment of nerve function: A clinical and experimental study. Acta Orthop Scand, 1967; Suppl 108: 1-155.

42. Hueston JT. Dupuytren's contracture and specific injury. Med J Aust, 1968; 1: 1084-5.

43. Stewart HD, Innes AR, Burke FD. The hand complication of Colles' fractures. J Hand Surg B, 1985; 10: 103-6.

44. Cooney WP, Dobyns JH, Lindscheid RL. Complications of Colle's fractures. J Bone Joint Surg A, 1980; 62: 613-9.

45. Kelly SA, Burke FD, Elliot D. Injury of the distal radius as a trigger to the onset of Dupuytren's disease. J Hand Surg B, 1992; 17: 225-9.

46. Kozin SH, Wood MB. Early soft-tissue complications after distal radius fractures. AAOS, Instr Cour Lect, 1993; 42: 8998.

47. Lanzetta M, Morrison WA. Dupuytren's disease occurring after a surgical injury to the hand. J Hand Surg B, 1996; 21: 481-3.

48. Hueston JT. Overview of etiology and pathology. En: Hueston JT, Tubiana R (Eds). Dupuytren's Disease, 2nd ed, Edinburgh: Churchill Livingstone, 1985; 75-81.
49. Thurston AJ. Dupuytren's disease. Review article. J Bone Jonit Surg B, 2003; 85: 469-77.

50. Hueston JT. The incidence of Dupuytren's contracture. Med J Aust, 1960; 47: 999-1002.

51. Hueston JT. Dupuytren's contracture: medicolegal aspects. Med J Aust, 1987; 147: S2S11.

52. Legueu F, Juvara E. Des aponévroses de la paume de la main. Bull Soc Anat, Paris, 1892; 5s 6: 383-400.

53. Merle M, Dautel G, Loda G. Lesiones de los tendones flexores. En: Mano Traumática. Urgencias. Barcelona: Masson SA, 1993: 179-96.

54. Sherman PJ, Lane LB. The palmar aponeurosis pulley as a cause of trigger finger. A report of two cases. J Bone Joint Surg A, 1996; 78: 1753-4.

55. Kaplan EB. Functional and Surgical Anatomy of the Hand. 2 ed. Philadelphia and Montreal: JB Lippincott Co, 1965: 251-2.

56. Powell BWEM, McLean NR, Jeffs JV. The incidence of a palmaris longus tendon in patients with Dupuytren's disease. J Hand Surg B, 1986; 11: 382-4.

57. McGrouther DA. The microanatomy of Dupuytren's contracture. Hand, 1982; 14: 215-36.

58. Tubiana R, Thomine JM, De Frenne $\mathrm{H}$. L'aponévrose palmaire et la maladie de Dupuytren. En: Tubiana R (Ed). Traité de Chirurgie de la Main, vol. 6. Paris: Masson, 1998: 30-49.

59. Kaplan EB. Functional and Surgical Anatomy of the Hand. Philadelphia: JB Lippincott Co, 1953: 247-52.

60. Stack HG. Muscle function in the fingers. J Bone Joint Surg B, 1962; 44: 899-909.

61. Gosset J. Anatomie des aponévroses palmo-digitales. En: Tubiana R, Hueston JT Eds). La Maladie de Dupuytren. Monographie du GEM, Paris: Expansion Scientifique Française, 1986; 20-31.

62. McGrouther DA. Anatomie microscopique de la maladie de Dupuytren. En: Tubiana R, 
Hueston JT (Eds). La Maladie de Dupuytren. Monographie du GEM, Paris: Expansion Scientifique Française, 1986; 32-48.

63. Tonkin MA, Burke FD, Varian JPW. The proximal interphalangeal joint in Dupuytren's disease. J Hand Surg B, 1985; 10: 358-64.

64. McFarlane RM. Anatomie de la maladie de Dupuytren. En: Tubiana R, Hueston JT (Eds). La Maladie de Dupuytren. Monographie du GEM, Expansion Scientifique Française, Paris, 1986; 49-63.

65. Bilderback KK, Rayan GM. Dupuytren's cord involving the septa of Legueu and Juvara: a case report. J Hand Surg A, 2002; 27: 344-6.

66. Thomine JM. Conjunctif d'enveloppe des doigts et squelette fibreux des commissures interdigitales. Ann Chir Plast, 1965; 10: 194-203.

67. Albinus BS. Historia Musculorum Hominis. Liber III, Cap CLXIV. Leidae Batavorum (Leiden): Theodorum Haak \& Henricum Mulhovium, 1734; 4725.

68. Skoog T. Dupuytren's contracture: Pathogenesis and surgical treatment. Surg Clin North Amer, 1967; 47: 433-44.

69. Skoog T. The transverse elements of the palmar aponeurosis in Dupuytren's contracture. Scand J Plast Surg, 1967; 1: 51-63.

70. Grapow M. Die Anatomie und physiologische Bedeutung der Palmaraponeurose. Arch Anat Physiol (Leipzig), Anat Abtheil, 1887; 2-3: 143-58.

71. Gosset J. Maladie de Dupuytren et anatomie des aponévroses palmo-digitales. Ann Chir, 1967; 21: 554-65.

72. Weitbrecht J. Ligamenta artuum superiorum sectio secundum, in syndesmologia sive historia ligamentorum corporis humani. Petropolis: Ex Typographia Acadamiae Scientarum, 1742: 43-4, 50.

73. Zancolli EA. Structural and Dynamic Bases of Hand Surgery,
Philadelphia: JB Lippincott, 1979; 3-36.

74. Mackenney Carrasco E. Dupuytren Radial. Estudio anatomo-clínico. Tesis Doctoral. Facultad De Medicina. Universidad de Alicante, 1995: 190-8.

75. De Frenne HA. Les structures aponévrotiques au niveau de la première commissure. Ann Chir, 1977; 31: 1017-9.

76. Tubiana R, Simmons BP, De Frenne HAR. Location of Dupuytren's disease on the radial aspect of the hand. Clin Orthop, 1982; 168: 222-9.

77. Tubiana R, De Frenne $\mathrm{H}$, Simmons B. Localisations de la maladie de Dupuytren à la partie radiale de la main. En: Tubiana R, Hueston JT (Eds). La Maladie de Dupuytren. Monographie du GEM, Paris: Expansion Scientifique Française, 1986: 191-7.

78. Kanavel AB. The thenar space. En: Infections of the Hand, Philadelphia: Lea \& Febiger, 1925: 133-40.

79. Grodinsky M, Holyoke EA. The fasciae and fascial spaces of the palm. Anat Rec, 1941; 79: 435-51.

80. Stanisavljevic S, Pool R. The paratendinous apparatus of the digits. J Bone Joint Surg B, 1962; 44: 910-2.

81. Larsen RD. Contractura de Dupuytren. En: Flynn JE (Ed). Cirugía de la Mano, Barcelona: Ed Toray, 1977; 691-724.

82. McFarlane RM. Patterns of the diseased fascia in the fingers in Dupuytren's contracture displacement of the neurovascular bundle. Plast Reconstr Surg, 1974; 54: 31-44.

83. Grayson J. The cutaneous ligaments of the digits. J Anat, 1941; 75: 164-5.

84. Cleland J. On the cutaneous ligaments of the phalanges. J Anat Phys, 1877-1878; 12: 526-7, plate XVII.

85. McFarlane RM. The finger. En: McFarlane RM, McGrouther DA, Flint MH (Eds). Dupuytren's Disease. Edinburgh: Churchil Livingstone, 1990: 155-67.
86. Bunnell S. Surgery of the intrinsic muscles of the hand. J Bone Joint Surg, 1942; 24: 1-31.

87. Landsmeer JMF. The anatomy of the dorsal aponeurosis of the human finger and its fonctional significance. Anat Rec, 1949: 104: 31-44.

88. Milford L. Les ligaments rétinaculaires et les amarres cutanées des doigts. En: Tubiana $R$ (Ed). Traité de Chirurgie de la Main, vol. 1. Paris: Masson, 1980: 267-70.

89. Thomine JM. La fascia digital. Développement et anatomie. En: Tubiana R, Hueston JT (Eds). La Maladie de Dupuytren. Monographie du GEM, Paris: Expansion Scientifique Française, 1986: 11-9.90. Thomine JM. La fascia digital. Développement et anatomie. En: Tubiana R, Hueston JT (Eds). La Maladie de Dupuytren. Monographie du GEM, Paris: Expansion Scientifique Française, 1972: 11-22.

91. Gosset J. Anatomie des aponévroses palmo-digitales. En: Tubiana R, Hueston JT (Eds). La Maladie de Dupuytren. Monographie du GEM, Paris: Expansion Scientifique Française: 1972: 23-37.

92. Milford LW Jr. Retaining ligaments of the digits of the hand. Gross and microscpic anatomic study. Philadelphia Co: WB Saunders, 1968: 23-4.

93. Law P, McGrouther DA. The dorsal wrinkle ligaments of the proximal interphalangeal joint. J Hand Surg B, 1984; 9: 271-5.

94. MacCallum Sir P, Hueston JT. The pathology of Dupuytren's contracture. Aust NZ J Surg, 1962; 31: 241-53.

95. Goyrand JGB. Nouvelles recherches sur la rétraction permanente des doigts. Gaz Méd de Paris, 1834; 2s 2: 219.

96. Hueston JT. Dupuytren's contracture: Comment on Dupuytren's contracture: Anatomy and pathogenesis of the digital cords and nodules. Hand Clin, 1991; 7: 659-60. 
97. Qureshi FI, Hornigold R, Spencer JD, Hall SM. Langerhans cells in Dupuytren's contracture. J Hand Surg B, 2001; 26: 362-7.

98. Sugden P, Andrew JG, Andrew SM, Freemont AJ. Dermal dendrocytes in Dupuytren's disease: A link betwenn the skin and pathogenesis?. J Hand Surg B, 1993; 18: 662-6.

99. Murrell GAC, Francis MJO, Bromley L. The collagen changes of Dupuytren's contracture. J Hand Surg B, 1991; 16: 2636.

100. Kischer CW, Speer DP. Microvascular changes in Dupuytren's contracture. J Hand Surg A, 1984; 9: 58-62.

101. Murrell GAC, Francis MJO, Howlett CR. Myofibroblast are not specific to Dupuytren's disease. En: Berger A, Delbrück A, Brenner P, et al. (Eds). Dupuytren's Disease. Berlin: Springer Verlag, 1994: 24-33.

102. Lennox IAC, Murali SR, Porter R. A study of the repeatability of the diagnosis of Dupuytren's contracture and its prevalence in the grampian region. J Hand Surg B, 1993; 18: 258-61.

103. Tubiana R, Michon J. Évaluation chiffré précise de la déformation dans la maladie de Dupuytren. Sa valeur pronostique. Mém Acad Chir, 1961; 87: 886-8.

104. Tubiana R. La maladie de Dupuytren. Étude clinique. Évaluation des lésions. En: Tubiana $R$ (Ed). Traité de Chirurgie de la Main, vol. 6. Paris: Masson, 1998: 53-66.

105. Erdmann MWH, Quaba AA, Sommerlad BC. Epithelioid sarcoma masquerading as Dupuytren's disease. Br J Plast Surg, 1995; 48: 39-42.

106. Chatelain D, Ouaknine M, De Pinieux G, et al. Localisation palmaire d'un sarcome épithélioïde simulant une maladie de Dupuytren. Rev Chir Orthop, 1999; 85: 731-4.

107. Hueston JT. The Dupuytren's diathesis. En: Dupuytren's Contracture. Baltimore: Williams and Wilkins Company, 1963: 51-63.

108. Hindocha S, John S, Stanley JK, et al. The heritability of Dupuytren's disease: familial aggregation and its clinical significance. J Hand Surgery A, 2006; 31: 204-10.

109. Paget J. On the minor signs of gout in the hands and feet. $\mathrm{Br}$ Med J, 1875; 1 (751): 665-6.

110. Garrod AE. On an unusual form of nodule upon the joints of the fingers. St Bartolomew's Hosp Rep, 1893; 29: 157-61.

111. Garrod AE. Concerning pads upon the finger joints and their clinical relationships. Br Med J, 1904; 2: 8.

112. Jones HW. Two cases of "knuckle pads". Br Med J, 1923; 1: 759.

113. Caroli A, Zanasi S, Marcuzzi $A$, et al. Epidemiological and structural findings supporting the fibromatous origin of dorsal knuckle pads. J Hand Surg B, 1991; 16: 258-62.

114. Mikkelsen OA. Knuckle pads in Dupuytren's disease. Hand, 1977; 9: 301-5.

115. Hueston JT. Some observations on knuckle pads. J Hand Surg B, 1984; 9: 75-8.

116. Hueston JT. Dorsal Dupuytren's disease J Hand Surg A, 1982; 7: 384-7.

117. Iselin F, Cardenas-Baron L, Gouget-Audry I, et al. La maladie de Dupuytren dorsale. Ann Chir Main, 1988; 7: 247-50.

118. Iselin M, Iselin F. Traité de Chirurgie de la Main. Paris: Ed Med Flammarion, 1967: 678.

119. Dupuytren G. De la rétraction des doigts par suite d'une affectation de l'aponeurose palmaire - description de la maladie - operation chirurgicale qui convient dans ce cas. Compte rendu de la clinique chirurgicale de l'Hôtel-Dieu par MM les Docteurs Alexandre Paillard et Marx. J Univ Hebd Méd Chir Pract Inst Méd, 1831, 5: 349-65.

120. Dupuytren G. De la rétraction des doigts et du diagnostic différentiel. En: Leçons orales de clinique chirurgicale faites à l'Hôtel-Dieu de Paris, Recuillies et publiées par MM les docteurs Brierre de Boismont et Marx, Deuxième édition entièrement refondue, vol. 4. Paris: Germer Baillière, 1839; 473502.

121. Ledderhose G. Zur Pathologie der Aponeurose des Fusses und der Hand. Arch Klin Chir, 1897; 55: 694-712.

122. Gordon SD. Dupuytren contracture: plantar involvement. Br J Plast Surg, 1964; 17: 4213.

123. Haedicke GJ, Sturim HS. Plantar fibromatosis: An isolated disease. Plast Reconstr Surg, 1989; 83: 296-300.

124. Classen DA, Hurst LN. Plantar fibromatosis and bilateral flexion contractures: a review of the literature. Ann Plast Surg, 1992; 28: 475-8.

125. Donato RR, Morrison WA. Dupuytren's disease in the feet causing flexion contracture in the toes. J Hand Surg B, 1996; 21: 364-6.

126. Kulkarni M, Elliot D. Dupuytren's disease of the big toe. J Hand Surg B, 2005; 30: 567-9.

127. De la Peyronie F. Sur quelques obstacles qui s'opposent à l'éjaculation naturelle de la semence. Mém Acad Royal Chir, 1743; vol 1, secc III, cap 19: 425-34.

128. Smith SP, Devaraj VS, Bunker TD. The association between frozen shoulder and Dupuytren's disease. J Shoulder Elbow Surg, 2001, 10: 149-51.

129. Bunker TD, Esler CNA. Frozen shoulder and lipids. J Bone Joint Surg B, 1995; 77: 684-6.

130. Rodeo SA, Hannafin JA, Tom $J$, et al. Immunolocalization of cytokines and their receptors in adhesive capsulitis of the shoulder. J Orthop Res, 1997; 15: 427-36.

131. Naylor IL, Coleman DJ, Coleman RA, ET AL. Reactivity of nodular cells in vitro: a guide to the pharmacological treatment 
of Dupuytren's contracture. En: Berger A, Delbrück A, Brenner P, ET Al. (Eds). Dupuytren's Disease. Berlin: Springer Verlag, 1994: 139-50.

132. Hurst LC, Badalamente MA. Nonoperative treatment of Dupuytren's disease. Hand Clin, 1999; 15: 97-107.

133. Pittet B, Rubbia-Brandt L, Desmoulière $A$, et al. Effect of 凶-Interferon on the clinical and biologic evolution of hypertrophic scars and Dupuytren's disease: An open pilot study. Plast Reconstr Surg, 1994; 93: 1224-35.

134. Dumontier C. La Maladie de Dupuytren. Origine, épidémiologie, étiologie et étiopathogénie. En: Tubiana $\mathrm{R}(\mathrm{Ed})$. Traité de Chirurgie de la Main, vol. 6. Paris: Masson, 1998: 8-30.

135. Starkweather KD, Lattuga S, Hurst LC, et al. Collagenase in the treatment of Dupuytren's disease: an in vitro study. J Hand Surg A, 1996; 21: 490-5.

136. Hurst LC, Badalamente MA Hentz VR, et al. Injectable collagenase clostridium histololyticum for Dupuytren's contracture. N Engl J Med, 2009; 361: 968-79.

137. Gilpin D, Coleman S, Hall S, et al. Injectable collagenasa clostridium histolyticum: a new nonsurgical treatment for Dupuytren's disease. J Hand Surg Am, 2010; 35: 2027-2038.e1.

138. Badalamente MA, Hurst LC Enzyme injection as nonsurgical treatment of Dupuytren's disease. J Hand Surg A, 2000; 25: 629-36.

139. Badalamente MA, Hurst LC, Hentz VR. Collagen as a clinical target: nonoperative treatment of Dupuytren's disease. J Hand Surg A, 2002; 27: 788-98.
140. Badalamente MA, Hurst LC. Efficacy and safety of injectable mixed collagenase subtypes in the treatment of Dupuytren's contracture. J Hand Surg Am, 2007; 32: 767-74.

141. Watt AJ, Curtin CM, Hentz VR. Collagenase injection as nonsurgical treatment of Dupuytren's disease: 8-year follow-up. J Hand Surg Am, 2010; 35: 534-9.

142. Daupleix D, Badois F, Dreyfus P. Affections dégénératives de la main et du poignet. Encycl Méd Chir (Paris), Thérapeutique, 25187 F10, 3-1987, 3p.

143. Badois FJ, Lermusiaux JL, Massé C, et al. Traitement chirurgical de la maladie de Dupuytren par aponévrotomie à l'aiguille. Rev Rhum, 1993; 60: 808-13.

144. Foucher G, Medina J, Navarro R. L'aponévrotomie percutanée à l'aiguille. Complications et résultats. Chir Main, 2001; 20: 206-11.

145. Tubiana R, Thomine JM. Le traitement chirurgical de la maladie de Dupuytren: technique des aponévrotomies et des aponévrectomies. En: Tubiana R, Hueston JT (Eds). La Maladie de Dupuytren. Monographie du GEM, Paris: Expansion Scientifique Française, 1972: 111-20.

146. Leclercq C. La Maladie de Dupuytren. Traitements non chirurgicaux. En: Tubiana $\mathrm{R}(\mathrm{Ed})$. Traité de Chirurgie de la Main, vol. 6. Paris: Masson, 1998; 6770.

147. Hueston JT. The table top test. Med J Aust, 1976; 2: 189-90.

148. Hueston JT. The table top test. Hand, 1982: 14: 100-3.

149. Smith AC. Dupuytren's contracture: Diagnosis and indi- cations for surgical treatment. Hand Clin, 1991; 7: 635-42.

150. Tubiana R. Surgical indications. En: Tubiana R, Leclercq C, Hurst LC, et al. (Eds). Dupuytren's Disease. London: Martin Dunitz, 2000; 218-22.

151. McGrouther DA. Dupuytren's contracture. En: Green DP, Hotchkiss RN, Pederson WC et al. (Eds). Green's Operative Hand Surgery. Philadelphia: Elsevier Churchill Livingstone, 2005: 159-85.

152. Hueston JT. Dermofasciectomy. En: McFarlane RM, McGrouther DA, Flint MH (Eds). Dupuytren's Disease. Edinburgh: Churchill Livingstone, 1990: 324-8.

153. Roy N, Sharma D, Mirza AH, et al. Fasciectomy and conservative full thickness skin grafting in Dupuytren's contracture. The fish technique. Acta Orthop Belg, 2006; 72: 678-82.

154. Leclercq C. La Maladie de Dupuytren. Les récidives. En: Tubiana $\mathrm{R}(\mathrm{Ed})$. Traité de Chirurgie de la Main, vol. 6. Paris: Masson, 1998: 108-16.

155. McGregor IA. Traitement de la maladie de Dupuytren par aponévrotomie et greffe. En: Tubiana R, Hueston JT (Eds). La Maladie de Dupuytren. Monographie du GEM. Paris: Expansion Scientifique Française, 1986; 165-71.

156. Hueston JT. Regression of Dupuytren's contracture. J Hand Surg B, 1992; 17: 453-7.

157. Tubiana R. Traitement des récidives. En: Tubiana R, Hueston JT (Eds). La Maladie de Dupuytren. Monographie du GEM. Paris: Expansion Scientifique Française, 1986: 14953. 